\title{
Modelos de evaluación institucional: el caso de la licenciatura de Sociología
}

Antonio Trinidad Requena*

Diego Gallego Martínez**

Luis Ayuso Sánchez**

Margarita Pérez Sánchez ${ }^{* * *}$

"...mi propósito no es el enseñar aquí el método que cada cual debe seguir para dirigir bien su razón, sino sólo el exponer de qué manera he tratado de conducir la mía. R. Descartes.

\section{Introducción}

A partir de los cambios llevados a cabo en materia educativa en muchos países europeos, se han diseñado diversas formas de evaluación universitaria, con el objetivo de conseguir una mayor racionalidad en el uso de los recursos asignados a la educación superior. Estos medios empezaron a materializarse en España a partir del Modelo Europeo de Evaluación, con el fin último de buscar puntos comunes en el proceso de evaluación para las diversas universidades europeas. Se tomó conciencia de que era necesario elaborar acciones orientadas a la mejora de la calidad universitaria y así adaptarse a un nuevo contexto educativo, tanto nacional como europeo. Gracias al impulso del Consejo de Universidades se puso en marcha el Programa Experimental de Evaluación de la Calidad Universitaria (1992-1994), que a pesar de ser una experiencia piloto ofreció resultados muy valorados por la comunidad universitaria.

Esta apuesta por la mejora de la calidad en las instituciones de educación superior queda patente en la elaboración de un
Plan Nacional de Evaluación de la Calidad de las Universidades (en adelante PNECU), publicado en 1995, y que tenía como objetivos: racionalizar la inversión, equiparar la Universidad española al contexto europeo, y dar información objetiva para llevar a cabo una toma de decisiones más eficiente. La intención es que la Universidad se conozca a sí misma, es decir, que sea capaz desde su autonomía de detectar cuáles son sus puntos fuertes y débiles y con ello desarrollar planes estratégicos que consigan la excelencia, la eficacia y la calidad, para así responder de una manera más adecuada al servicio que presta a la sociedad.

Con esta filosofía se lleva a cabo la segunda convocatoria del PNECU (1998) a la que concurrieron 51 universidades que correspondían a 230 unidades evaluadas. La práctica totalidad de ellas presentaron informes de autoevaluación (94\%) y evaluación externa (92\%), y algo más de la mitad realizaron informes finales de titulación, mientras que sólo un $27 \%$ llevó a cabo el Informe Final de Universidad. Los resultados obtenidos fueron relativamente satisfactorios para el Consejo de Universidades, aunque se puso de manifiesto que la evaluación puede ser entendida de diversas maneras, ejemplo de ello es este estudio, que tiene como objetivo comparar tres experiencias de evaluación realizadas en distintas titulaciones de Sociología.

Las unidades evaluadas objeto de análisis son las licenciaturas de Sociología de Deusto, Navarra y Granada. El equipo de trabajo estimó oportuno para comparar estas evaluaciones tomar 
como referencia la propuesta del Consejo de Universidades, por el consenso generalizado de la utilidad de esta metodología. Lo que se pretendía era conocer como se habían llevado a cabo los procesos de autoevaluación y la metodología seguida en cada uno de ellos, para establecer así comparaciones y enriquecer los indicadores propuestos por la Guía de Evaluación. Para que la comparación fuera exhaustiva y comprensible, se tomaron como referencia los vaciados propuestos por el Consejo de Universidades, que tenían como objetivo presentar de forma esquemática un resumen de todos los indicadores que debían analizarse, describirse y valorarse para conocer mejor la realidad universitaria.

Tal y como recomienda el PNECU, los equipos de evaluación pueden utilizar todo tipo de herramientas metodológicas e indicadores para conocer de manera más real la situación en la que se encuentran las licenciaturas. De ahí que las dimensiones deban ser interpretadas de manera flexible, pues como bien recogen diversos autores los procesos de evaluación han de estar en relación directa con el contexto, es decir, han de conocer las características singulares de la comunidad y de sus participantes, con el objetivo de hacer una valoración global del objeto a evaluar, señalando sus puntos fuertes y débiles, y el ajuste de sus metas y prioridades (STUFFLEBEAM, 1971).

Debido a la heterogeneidad de situaciones en las que se encuentran las universidades españolas, y al diferente criterio con el que se puede interpretar la Guía de evaluación, este trabajo pone de manifiesto cómo a través de diversas herramientas y técnicas evaluativas se puede conseguir un fin parecido: conocer mejor la realidad de cada Facultad, tomar conciencia de la misma y llevar a cabo estrategias de mejora. Por otro lado, la novedad que presenta esta investigación es la comparación metodológica de evaluaciones. Conocer qué técnicas se han utilizado y cómo se han usado las herramientas, ayuda a conocer mejor los diferentes enfoques que existen de la evaluación educativa y permite establecer un necesario consenso metodológico entre los indicadores más adecuados para la evaluación de las instituciones universitarias.

\section{Titulaciones evaluadas}

Las universidades escogidas para el estudio presentan un contexto muy diferenciado. Por un lado, se encuentra la licenciatura de Sociología de la Universidad de Navarra, que tiene un carácter público, al igual que la de Granada, aunque las diferencias son significativas y van desde el contexto económico hasta el cultural. Junto a estas dos se analiza la licenciatura de Sociología per- teneciente a la Universidad privada de Deusto, con un carácter confesional. Antes de comentar brevemente cómo han realizado sus procesos de autoevaluación, hay que recordar que sólo Navarra sigue de forma más o menos lineal las directrices propuestas por la Guía de Evaluación del PNECU. Las otras dos titulaciones han seguido modelos "propios", así, Deusto ha elaborado un informe que se sustenta en tres pilares (entorno, desarrollo de la docencia y la investigación, y clima corporativo), apareciendo muchos temas mezclados. En último lugar destaca Granada, que aporta una visión holística de la evaluación, y donde se han determinado las dimensiones y subdimensiones del proceso a partir de la negociación entre los miembros que formaban parte de este centro.

El Informe presentado por la licenciatura de Sociología de la Universidad de Navarra se termina en abril del 2000. Para su elaboración se creo el Comité de Autoevaluación de Sociología y Trabajo Social, compuesto por tres directores de departamento junto con los profesores más antiguos y de mayor prestigio. Igualmente, y con el objetivo de recoger y procesar la información se crearon dos subcomités de enseñanza, correspondientes a los respectivos departamentos donde estaban representados todos los miembros de la Facultad. En lo que se refiere a cuestiones metodológicas y de contenido, se han utilizado los métodos recomendados por la Guía de Evaluación, tanto técnicas cualitativas como cuantitativas, aunque se observa un mayor peso de estas últimas. Del mismo modo, resalta la preponderancia de la enseñanza sobre la investigación y la gestión, que apenas aparece a lo largo del informe, por su excesiva centralización según el propio comité.

La estructura interna del informe se divide en diez apartados, que se complementan con un anexo de tablas elaboradas a partir de los indicadores utilizados y que se proponen en la Guía de Evaluación. El primero de estos apartados evalúa el contexto, donde se trata la situación que ocupa esta licenciatura dentro de la Universidad de Navarra, se presenta la estructura interna de la misma y las funciones que cada miembro debe desempeñar. Del mismo modo, también se aporta la trayectoria desde su fundación y el perfil de los egresados. El segundo de los puntos analiza las metas y objetivos de la institución, realizando una valoración general de los mismos, centrándose sobre todo en la plasmación de éstos en el plan de estudios, las posibles salidas que pueden encontrar los licenciados en el mercado de trabajo, o los desafíos futuros fijados que debe abordar la titulación en busca de una mejora continua de la calidad.

En tercer lugar, y tal como recomienda la Guía de Evaluación, se analiza el programa de formación. En este apartado se recogen opiniones de los diversos miembros implicados en la titulación sobre cuestiones como la distribución de la carga docente, los horarios, los contenidos y programas de las asignaturas, e incluso la motivación del alumnado. El desarrollo de la 
enseñanza es analizado por medio del funcionamiento y la práctica de las tutorías, el análisis de la metodología docente, las estrategias didácticas o la coordinación entre profesores. En un plano más cuantitativo, se presentan los resultados de la enseñanza con el objetivo de aportar cifras sobre el rendimiento, el éxito o el fracaso. Junto a esto, se presentan otra serie de datos más relacionados con la experiencia y la visión del propio estudiante; como el número de veces que van a tutorías, o el número de horas de estudio a la semana. Los indicadores utilizados para evaluar a los alumnos pueden dividirse en dos: en primer lugar, aquellos relacionados con aspectos más burocráticos, como el número de alumnos por curso; su evolución por años. En segundo lugar, aquellos aspectos menos formales, resaltando la participación del alumno en la vida universitaria.

Otro de los apartados hace referencia a los recursos humanos y a las instalaciones. Para el primero de ellos, se utilizan dimensiones como la formación adquirida y su categoría docente, combinándose estas con otras variables. En cuanto al segundo de los aspectos, el informe utiliza indicadores como la dotación de materiales e infraestructuras. Toda esta información se completa con valoraciones que sobre este tema han realizado sus usuarios, analizándose prácticamente todas las instalaciones de las que disfruta la Facultad. En la evaluación de las relaciones externas se estudia la presencia del centro en el extranjero a través de la movilidad tanto de estudiantes como de profesores, además se refleja una especial preocupación por la imagen de la licenciatura en la sociedad Navarra y se valora la presencia de convenios o colaboraciones con ayuntamientos, sindicatos, asociaciones y empresas. Esta evaluación de la enseñanza termina con la elaboración de puntos débiles y fuertes; las principales fortalezas a las que se hace referencia ascienden a doce, y el número de debilidades a veintidós. Concluye con propuestas de acciones de mejora y recomendaciones dirigidas a la Facultad y al Departamento (24), así como al Vicerrectorado de Ordenación Académica Alumnos y Nuevas Tecnologías (12).

En lo que se refiere a la investigación, el informe realiza un balance de esta actividad tal y como propone la Guía de Evaluación, aunque presenta más deficiencias que la enseñanza. El comité repasa la actividad investigadora llevada a cabo durante el período 1994-1998, reflejando los puntos fuertes y débiles que se han encontrado. En lo que se refiere a debilidades se destacan las limitaciones tanto cualitativas como cuantitativas existentes en el Departamento, y en cuanto a fortalezas se muestra una especial satisfacción por la importante dedicación de los profesores, así como las vinculaciones institucionales que poco a poco va alcanzando la unidad evaluada. En lo que respecta a la gestión, y como se menciono con anterioridad, el comité de autoevaluación omite su estudio.

La licenciatura de Sociología de la Universidad de Deusto en Bilbao parte con unas características diferentes a la anterior al considerarse un centro de carácter "confesional, privado y vasco". En su proceso de autoevaluación se han seguido unas directrices propias, usando sobre todo técnicas cuantitativas. Se presentaron los resultados en mayo de 2000. La estructura en la que se ha dividido este proceso esta compuesta por cuatro bloques. En primer lugar, el entorno sociopolítico y universitario donde se inserta la licenciatura. En segundo lugar, el desarrollo de la docencia, la investigación y la organización de los recursos. En tercer lugar, el clima corporativo que conforman todas las personas miembros de la Facultad. En cuarto y último lugar, se realiza una reflexión crítica para determinar cuales son los puntos fuertes y débiles del centro. Tras realizar todo el proceso, el comité elabora unas conclusiones y establece unas propuestas de acción.

En lo que se refiere al contexto, el informe destaca las características socioculturales y competitivas que afectan a la licenciatura por sus propias particularidades, y analiza la posición de la Facultad dentro de la Universidad y su historia reciente. Otro de los apartados evaluados hace referencia a los recursos materiales y humanos, ofreciéndose una visión general de carácter cuantitativo. Respecto a los recursos materiales apenas se ofrece información sobre los mismos, sin embargo, los recursos humanos son tratados de manera más amplia y completa, utilizando para ello fuentes secundarias y diversos indicadores. El análisis se estructura en torno al profesorado, combinando cuestiones como la edad, la formación, o la metodología docente y al alumnado, considerándose su número y la evolución de los últimos años.

El segundo apartado hace referencia a la estructura de la Facultad, donde se parte de la diferencia entre los dos planes de estudios vigentes y sus correspondientes programas de investigación y formación. Posteriormente, se evalúa la docencia y se mezclan diferentes aspectos como el desarrollo de la enseñanza y los objetivos de la misma. En estos últimos, el proceso debe detenerse con mayor rigor para determinar si han sido alcanzados (SCRIVEN, 1967). El comité aplica un amplio número de indicadores, que van desde los más clásicos (distribución de la carga lectiva), hasta los más novedosos y adaptados al contexto (oferta multilingüística), sin olvidar los relacionados con la metodología, como la orientación práctica de los futuros egresados.

La investigación es otro de los puntos que se trata en este apartado, analizando los grupos de investigación, las publicaciones, la participación en proyectos, etc. Sobresale la inexistencia de objetivos de $\mathrm{I}+\mathrm{D}$, la debilidad de proyectos de investigación y la falta de recursos económicos destinados a esta labor. En lo que respecta a la formación, nuevamente se hace referencia al profesorado y a los alumnos. De los primeros estudia todo lo relacionado con su formación contínua, la metodología docente, o la forma de evaluar los conocimientos adquiridos en las distintas asignaturas por parte de los alumnos. En cuanto a los estudiantes se analizan temas como el número de horas lectivas, número de créditos por curso y la distribución de horarios. 
El tercer pilar del proceso de evaluación lo constituye el estudio del clima corporativo del centro. Para su análisis la comisión evaluadora estimó oportuno dividirlo en tres partes. La primera de ellas hace referencia a la gestión, la cual se organiza en torno a un sistema jerárquico de autoridad. En segundo lugar, se localiza la licenciatura dentro del entramado universitario de Deusto. Por último, y en un apartado titulado "clima organizacional", se refleja la participación de los profesores en las actividades relacionadas de forma directa con la institución y se ofrecen una serie de estrategias de acción para mejorar el ambiente del centro. El informe finaliza con unas conclusiones donde se reflejan las debilidades y fortalezas detectadas y se complementa con cuatro propuestas de acción presentadas a la dirección de la universidad para su "consideración, examen y potencial puesta en práctica".

El último proceso de evaluación analizado es el llevada a cabo en la licenciatura de CC.PP y Sociología de la Universidad de Granada, donde la experiencia de evaluación esta vigente desde el año 1992, gracias a los procesos de autoevaluación realizados por el Departamento de Sociología (TRINIDAD, 1995). Estas primeras experiencias evaluativas se caracterizaron por la negociación entre todos los miembros del centro de las dimensiones y los indicadores que mejor definían la calidad universitaria. Con este objetivo se suministró un cuestionario entre profesores, alumnos y equipos directivos para su delimitación. En el año 1998 se volvió a realizar otro estudio autoevaluativo con las mismas características que el anterior, aunque con algunos indicadores más y con la intención de comparar resultados. La visión holística que ha guiado este proceso es característico de las evaluaciones puestas en marcha en esta facultad, siendo la propia comunidad universitaria la que ha respondido a la clásica pregunta: ¿qué evaluar?

El informe presentado se estructuró en siete dimensiones objeto de análisis. Comienza con la evaluación del contexto, donde se discute la valoración que la comunidad universitaria hace de la presencia y relación institucional de la Facultad con la ciudad de Granada. Posteriormente se presta atención a los recursos, analizándose la opinión de alumnos y profesores sobre los medios económicos y materiales de la licenciatura, añadiendo una dimensión comparativa en el tiempo (1992-1998). Otra de las dimensiones evaluadas es el profesorado, donde se parte de la propia autoevaluación, ofreciendo resultados referidos a cantidad, calidad, satisfacción y coordinación de los mismos.

En cuarto lugar se estudia el alumnado, presentándose variables como los hábitos de estudio, la satisfacción con la carrera, o el perfil de los nuevos alumnos. A continuación se analiza la organización y el funcionamiento de la licenciatura, centrando la atención en la organización docente, el plan de estudios, los servicios y la toma de decisiones. También se tiene en cuenta al Rectorado, especialmente aquellos aspectos que directa o indirectamente inciden en el funcionamiento de la Facultad, como becas o apoyo a la investigación. La última dimensión recoge una valoración global de todos los actores implicados en la misma, comparando nuevamente sus resultados con los de 1992. Para terminar, y a modo de síntesis, se presentan unas conclusiones que se dividen según cada una de las dimensiones analizadas, sirviendo como propuestas para la mejora de la calidad docente, investigadora y organizativa.

El modelo de evaluación seguido por este comité tiene la intención de responder a unos objetivos predeterminados como: diseñar un tipo de evaluación adaptado a las peculiaridades de la Facultad, elaborar y validar las distintas técnicas de evaluación a utilizar, conocer la realidad educativa de la Facultad y formular juicios de valor que sirvan para mejorar la toma de decisiones. Para cumplir estos objetivos se siguen unas líneas teóricas basadas en el estamento del que surge la acción de evaluar (comunidad universitaria), decidiéndose por la evaluación interna, al dar capacidad a los centros para resolver sus problemas (HopKINS, 1985). Igualmente, se optó por una evaluación de carácter formativo, pues contribuye al perfeccionamiento de un programa en desarrollo que se realiza durante el proceso de enseñanzaaprendizaje, a través del cual se va constatando la validez de todos los componentes del proceso con respecto al logro de los objetivos que se pretenden (SCRIVEN, 1967).

El enfoque que siguió el comité de evaluación es global u holístico, al considerar que es el que mejor se adapta a un centro universitario y el que puede ser más completo (SimONS, 1985). En cuanto a las perspectivas metodológicas seguidas, esta investigación ha superado la tradicional dicotomía cualitativo-cuantitativo, optando por la complementariedad de ambas. Así, se realizaron cuestionarios de evaluación destinados a profesores, alumnos y licenciados. Igualmente, se llevaron a cabo siete grupo de discusión, participando todos los miembros que componen la realidad de la Facultad (exceptuando el PAS). Por otro lado, también se aplicaron entrevistas en profundidad no estructuradas (seis), que estaban dirigidas a personas que trabajaban en los distintos servicios del centro. Por último, se realizó una revisión documental a través de informes y documentos procedentes de la secretaría y biblioteca de la Facultad, así como de la base de datos de la Universidad de Granada. En cuanto al diseño, se ha seguido el estudio de casos, entendido como una investigación sistemática sobre una instancia específica.

Por tanto, este proceso de evaluación se ha dividido en dos partes. En un primer plano destaca el diseño de la evaluación y en un segundo la evaluación institucional. En ambas fases se ha tenido en cuenta una perspectiva comparada con el estudio anterior, realizado en 1992 en esta misma Facultad. Por último, destacar que la elección de dimensiones e indicadores se ha realizado teniendo en cuenta los resultados del primer estudio, la valoración de los alumnos de la Facultad, las directrices recogidas en la Guía de Evaluación del PNECU, y las recomendaciones del Consejo Europeo en materia de calidad de la enseñanza superior. 


\section{Comparación y análisis de los indicadores utilizados en los procesos de autoevaluación}

Tomando como referencia las indicaciones y los indicadores propuestos por el Plan Nacional de Evaluación, se han analizado los tres informes objeto de este estudio. El objetivo principal consiste en establecer puntos de comparación entre los indicadores y las herramientas metodológicas utilizadas, para así conseguir un estudio más profundo y enriquecedor sobre estas tres autoevaluaciones. Para ello, y buscando una mayor claridad y comprensión, se optó por establecer cuadros de síntesis de cada uno de los programas, donde se reflejasen las semejanzas y diferencias que los informes analizados tenían respecto al PNECU y entre ellos mismos. Gracias a esta metodología de síntesis se pudieron evaluar (metaevaluar) los estudios presentados, lo que permitió conocer en que medida las facultades habían seguido lo recomendado por el Consejo de Universidades, o si habían utilizado otras herramientas e indicadores. Por todo ello, este estudio opto por dividirse en las tres grandes dimensiones que recomienda el PNECU, es decir, enseñanza, investigación y gestión.

La enseñanza es el apartado que mejor ha sido tratado tanto cualitativa como cuantitativamente por los tres informes. En lo que respecta a los datos globales del contexto, sólo Granada se preocupa de evaluar la relación que la titulación tiene con el entorno socioeconómico donde se inserta la licenciatura, utilizando para ello cuestionarios administrados a profesores y alumnos, donde se analizan cuestiones como: la comunicación con el mercado laboral local, la información desde la Facultad al entorno, los estudios sobre la realidad cercana, e incluso la relación de algunas asignaturas con el propio contexto. Las otras dos facultades no analizan la estructura productiva y social sobre la que se sustenta la realidad universitaria, ni la aportación que ésta hace a aquella, sino que se detienen sobre todo en la posición de la titulación y en los órganos de gobierno, haciendo sólo una descripción de los mismos. A este respecto, resalta el análisis realizado por la Facultad de Deusto, que analiza el peso que la titulación tiene en el conjunto de la Universidad y ofrece una evolución, tomando como referencia su número de alumnos desde el año 1994. Por su parte, el comité de autoevaluación de Navarra se refiere al tema afirmando que: "la titulación está incluida en la Facultad de Ciencias Humanas y Sociales y comenzó a impartirse en el curso 1991-1992 con 121 alumnos. El número de estos que cursó estudios en 1998-1999 fue de 665, esto es, el 6,28\% del total de la Universidad y el 21,31\% de la Facultad de Ciencias Humanas y Sociales"
Los órganos que componen y sustentan la Universidad son tratados en estos informes de forma meramente descriptiva, limitándose a ofrecer pequeñas referencias sobre el esquema organizacional y la representatividad que se tiene en el mismo. Muy unido con este tema se encuentra la toma de decisiones que emerge de los órganos de gobierno, y que es tratado por las tres titulaciones. Navarra, no analiza las funciones y la toma de decisiones de los órganos, sino que hace un juicio de valor de los distintos estamentos. Por su parte, Deusto hace todo lo contrario, es decir, enumera y describe los órganos que intervienen en el diseño de la gestión y la toma de decisiones. Granada presenta valoraciones realizadas por profesores y alumnos sobre la toma de decisiones en la Facultad y en los departamentos, además estos datos son comparados con los de la evaluación realizada en 1992.

La última dimensión que recomienda el PNECU para estudiar el contexto es la historia y la evolución de las titulaciones, elemento clave para entender cualquier centro. Las facultades del norte hacen un pequeño recorrido tanto por sus años de existencia, como de los planes de estudios, aunque no ofrece ningún juicio de valor sobre la utilidad y la necesidad que tanto hoy como ayer tenía una titulación de dichas características. Muy distinto es el informe de Granada, que no presenta un estudio detallado de los cambios históricos de la licenciatura, justificado tanto por su corta historia, como por las contínuas alusiones que se realizan al primer informe.

Para el buen funcionamiento de cualquier organización es necesario tener unos objetivos y metas claros, pues la calidad se puede medir en función del grado de consecución de objetivos prefijados por la Universidad (PÉREZ VERA 1991). En general, el análisis refleja que existen unos objetivos muy generales y poco especificados en las facultades estudiadas (Cuadro 1). No se presenta en ninguno de los informes un apartado concreto donde se analice el proceso de revisión de objetivos. Según TyLer (1967), el proceso de evaluación en una institución educativa consiste, en primer lugar, en identificar los objetivos que la institución se ha propuesto para un período de tiempo concreto, en segundo lugar, en medir los resultados en el período marcado, y finalmente, contrastar hasta que grado se han logrado los objetivos propuestos. A pesar de la importancia que tienen los objetivos, sólo Deusto los presenta, aunque de manera muy general y sin revisar dichos objetivos. Por otro lado, en los tres informes se hacen continuas alusiones a los planes de estudio y a su revisión, esto puede ser interpretado como un replanteamiento de objetivos de la titulación, o como una nueva respuesta a las necesidades del mercado de trabajo.

Respecto a los indicadores relacionados con la implantación de la titulación, la Facultad de Navarra presenta un análisis de carácter interno; "el contexto en el que se implantó la licenciatura de Sociología se caracteriza por un esfuerzo de ampliación del número de titulaciones acompañada por una reforma global de los planes de 
estudio". Deusto, que cambió el Plan de estudios recientemente, se detiene en este apartado y valora los cambios efectuados motivados por el contexto tan competitivo en el que se inserta. La relación entre el mercado de trabajo y el Plan de estudios sólo es analizada de forma directa por Granada, que a través de los grupos de discusión recoge opiniones de licenciados y profesores. A este respecto, hay que destacar que Navarra cierra la cuestión afirmando que sus egresados "salen al mercado con un bagaje insuficiente para abordar con solvencia la práctica profesional de la Sociología".

En cuanto a la oferta de plazas destaca, el trato excesivamente descriptivo de estos indicadores, no usándose como medidores de calidad (J. VIDA, 2000), y sólo Deusto realiza una valoración del mismo, debido posiblemente a su carácter privado. Granada por su parte, establece perfiles de matriculación de los nuevos ingresados. Como última dimensión destaca la relación existente entre la demanda de sociólogos y la empleabilidad. Este apartado, es tratado de forma diversa; por un lado Deusto no presenta de manera directa una respuesta a este indicador, Navarra ofrece posibles campos de trabajo de los sociólogos, pero no realiza ningún estudio concreto sobre el empleo de sus titulados, y Granada aporta sólo las valoraciones de sus licenciados.

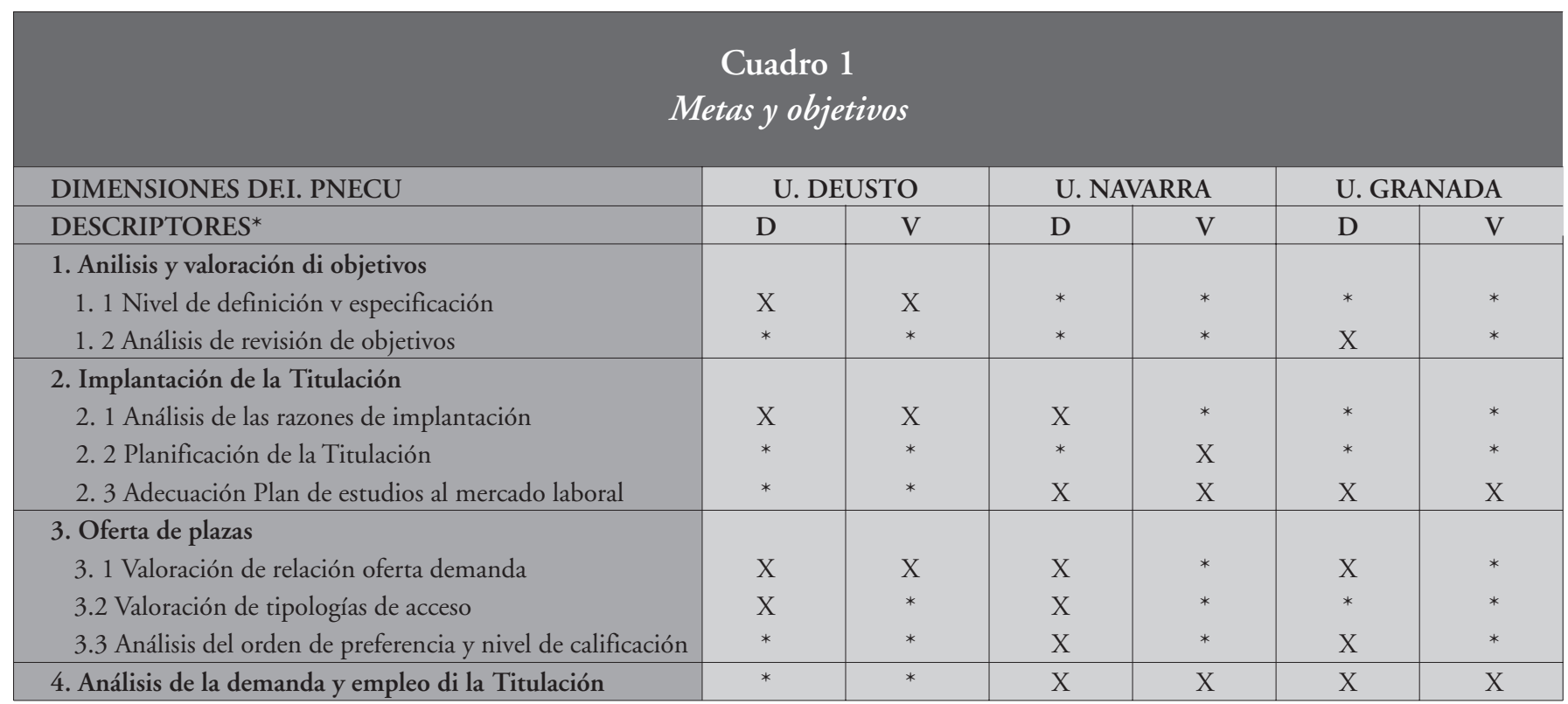

* Fuente: elaboración propia a partir de los vaciados del PNECU

* Leyenda: "X" el indicador ha sido tratado, "*” el indicador no ha sido tratado, "D" describe, "V" valora

El PNECU divide el apartado de la formación en tres bloques (Cuadro 2). En primer lugar valora la estructura del plan, en segundo lugar analiza los programas de las asignaturas, y un tercer apartado evalúa la organización de la enseñanza. En definitiva, lo que propone la Guía de Evaluación es un análisis completo del plan de estudios, pero esta evaluación se realiza sin tener en cuenta la posibilidad del departamento de impartir docencia en varias titulaciones (A. BOL, V. TRICIO, 2000). En cuanto a la estructura del plan de estudios - perfil de formación, directrices generales del título, dimensión práctica-, tanto Deusto como Navarra ofrecen valoraciones a partir de datos cuantitativos y tablas dirigidas a mejorar su sistema actual, mientras que Granada por medio de información cualitativa presenta valoraciones y recoge puntos débiles que deben ser mejorados. Así se puede concluir que, a través de distintas vías de recogida de información, se pueden cumplir las directrices del Plan Nacional de Evaluación de Universidades.

La segunda dimensión que se analiza en este bloque son los programas de las asignaturas. De los estudios consultados el que más se aproxima a lo exigido por el PNECU es el de Navarra, donde se presentan las tablas recomendadas por el mismo. La Facultad vasca no ofrece tablas tan pormenorizadas, aunque lo presentado es suficiente para responder a algunos indicadores. El estudio granadino sólo aporta una serie de reflexiones sobre algunos de los indicadores propuestos, como la valoración de los programas y la metodología docente. El primero de estos indicadores ha sido objeto de una especial atención. Navarra tras analizar los cuarenta y tres programas de las asignaturas, concluye "que por lo general se puede valorar muy positivamente la información 
que se transmite en los programas de las asignaturas, aún cuando se hecha en falta un esfuerzo por normalizar el diseño de los mismos". Del mismo modo, Deusto recoge esta valoración: "con mayor o menor amplitud la mayoría de los programas ofrecen información sobre el temario, especificando detalladamente los objetivos a lograr en dicho curso en un 70,5\%". La Facultad andaluza también se ha detenido en el análisis de este apartado, y concluye afirmando que "los contenidos estaban actualizados y hacían referencia a la realidad social más próxima”. En el apartado referido a la organi- zación de la enseñanza existen algunos indicadores que son tratados por todas las titulaciones, como los órganos responsables, el horario semanal de docencia o la planificación de exámenes finales; el resto son abordados sobre todo por Deusto y Navarra. Como aspecto novedoso resalta que Granada se preocupe del grado de satisfacción que tienen los alumnos sobre el funcionamiento del centro, lo que posibilita que el análisis sea más completo.

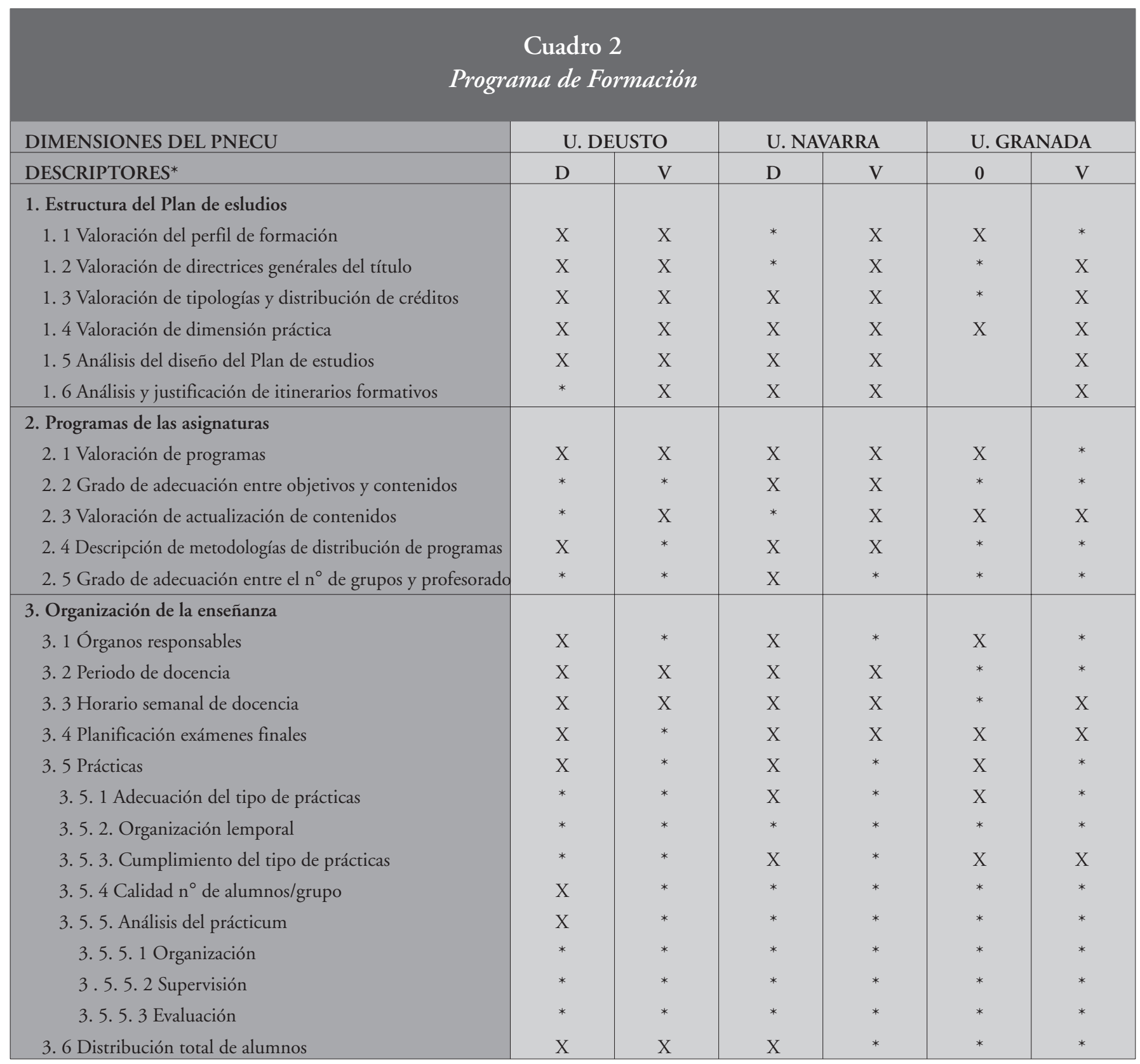

* Fuente: elaboración propia a partir de los vaciados del PNECU

* Leyenda: "X" el indicador ha sido tratado, "*” el indicador no ha sido tratado, "D" describe, "V" Valora" 
Muy relacionado con el programa de formación se encuentra el desarrollo de la enseñanza, que ha sido una de las dimensiones a la que más importancia le han dedicado estos informes (Cuadro 3). En lo que se refiere a la atención tutorial destaca sobre todo el carácter valorativo de Deusto y Navarra, que no está basado en evidencias, sino en opiniones y percepciones del comité evalua- dor. Granada, no utiliza indicadores relacionados con la atención tutorial, exceptuando el nivel de cumplimiento que se hace de las mismas. Tras el informe realizado por las tres facultades, parece haber un consenso claro: las tutorías se cumplen, pero los alumnos no saben utilizarlas (exceptuando la Facultad de Deusto).

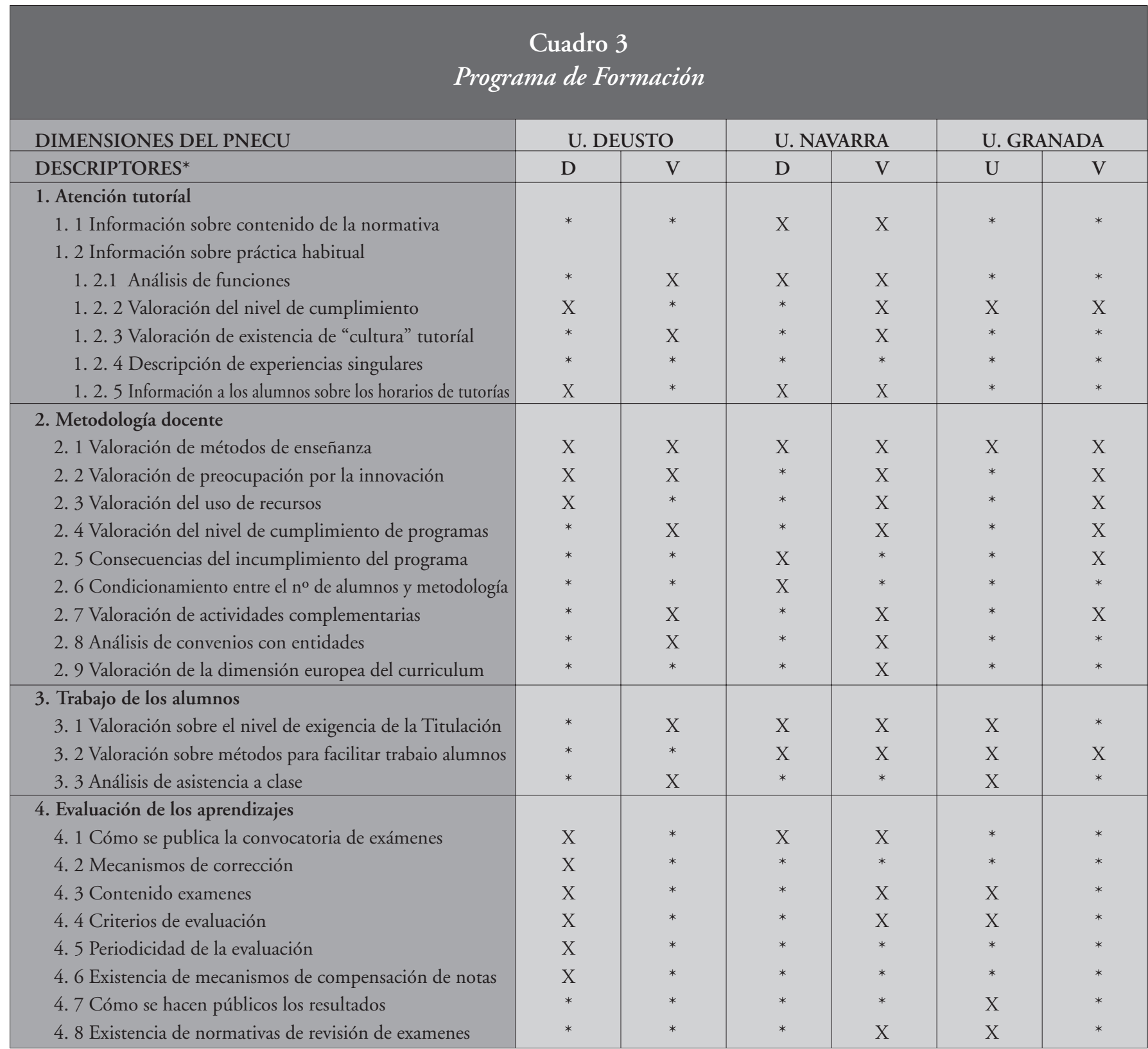

* Fuente: Elaboración propia a partir de los vaciados del PNECU

* Leyenda: "X" el indicador ha sido tratado, "*" el indicador no ha sido tratado, "D" describe, "V" valora

El análisis que han realizado los equipos de evaluación sobre la metodología docente, muestra que la información recogida ha sido sobre todo valorativa, aunque esta mayoritariamente se ha basado en evidencias. Los indicadores más tratados se corresponden con la valoración de los métodos de enseñanza, la preocupación por la innovación, el uso de recursos y el nivel de cumpli- 
miento de los programas. Uno de los aspectos que más resalta es la poca importancia dada a la formación no reglada, que a pesar de ser valorada por algunas facultades no es evaluada con todo el detenimiento que debiera. En cuanto al trabajo de los alumnos se observa una valoración general, aunque Granada presenta una serie de indicadores descriptivos con los que responder a este apartado. Los informes de Deusto y Navarra se adaptan a lo exigido por el PNECU, ya que ofrecen datos sobre las horas de clase, el tiempo de estudio, métodos de trabajo, etc., a partir de los cuales realizan sus valoraciones. El comité vasco hace una reflexión crítica sobre este apartado y afirma que hay "un calendario sobrecargado en una tarea de escucha escolar sobredimensiona- da... . La evaluación de los aprendizajes ha sido tratada por todos los informes, a pesar de ésto se aprecia una mayor tendencia a la descripción en Deusto y Granada, limitándose Navarra a la valoración de algunos indicadores.

Los conocimientos que se trasmiten en las aulas de las universidades han sido cuantificados desde que la institución existe (Cuadro 4). Por ello, una de las dimensiones que no debe faltar en la evaluación de la enseñanza universitaria es la que hace referencia a los resultados de la misma. Estos indicadores deben tener en cuenta la información contextual y los juicios y valoraciones de los agentes implicados (Cuenin, 1987), (FraCKMAMn, 1989),

\begin{tabular}{|c|c|c|c|c|c|c|}
\hline \multicolumn{7}{|c|}{$\begin{array}{c}\text { Cuadro } 4 \\
\text { Resultados de la enseñanza }\end{array}$} \\
\hline DIMENSIONES DLL PNECU & & & & RA & & \\
\hline DESCRIPTORES & $\mathrm{D}$ & $\mathrm{V}$ & $\mathrm{D}$ & $\mathrm{V}$ & $\mathrm{D}$ & $\mathrm{V}$ \\
\hline $\begin{array}{l}\text { 1. Indicadores de rendimiento global de la Titulación } \\
\text { 1. } 1 \text { Tasa de abandono en el primer año } \\
\text { 1. } 2 \text { Tasa de abandono en los dos primeros años } \\
\text { 1. } 3 \text { Tasa de presentados } \\
\text { 1. } 4 \text { Tasa de éxito } \\
\text { 1. } 5 \text { Tasa de rendimiento }\end{array}$ & $\begin{array}{l}\mathrm{X} \\
\mathrm{X} \\
\mathrm{X} \\
\mathrm{X} \\
*\end{array}$ & $\begin{array}{l}* \\
* \\
* \\
* \\
* \\
*\end{array}$ & $\begin{array}{l}* \\
* \\
\mathrm{X} \\
\mathrm{X} \\
\mathrm{X}\end{array}$ & $\begin{array}{l}* \\
* \\
* \\
\mathrm{X} \\
\mathrm{X}\end{array}$ & $\begin{array}{l}* \\
\mathrm{X} \\
\mathrm{X} \\
\mathrm{X} \\
\mathrm{X}\end{array}$ & $\begin{array}{l}* \\
* \\
* \\
* \\
* \\
*\end{array}$ \\
\hline $\begin{array}{l}\text { 2. Indicadores de graduación } \\
\text { 2. } 1 \text { Tasa de éxito } \\
\text { 2. } 2 \text { Tasa de retraso } \\
\text { 2. } 3 \text { Tasa de abandono } \\
\text { 2. } 4 \text { Duración media de los estudios }\end{array}$ & $\begin{array}{l}X \\
X \\
X \\
X\end{array}$ & $\begin{array}{l}* \\
* \\
* \\
*\end{array}$ & $\begin{array}{l}* \\
* \\
* \\
*\end{array}$ & $\begin{array}{l}* \\
* \\
* \\
*\end{array}$ & $\begin{array}{l}\mathrm{X} \\
\mathrm{X} \\
\mathrm{X} \\
*\end{array}$ & $\begin{array}{l}* \\
* \\
* \\
*\end{array}$ \\
\hline $\begin{array}{l}\text { 3. Valoración y análisis de resultados diferidos } \\
\text { 3. } 1 \text { Empleo y demanda de graduados } \\
\text { 3. } 2 \text { Opinión de los titulados sobre su formación } \\
\text { 3. } 3 \text { Opinión de empresarios sobre formación de titulados }\end{array}$ & $\begin{array}{l}* \\
* \\
*\end{array}$ & $\begin{array}{l}* \\
* \\
*\end{array}$ & $\begin{array}{c}* \\
\mathrm{X} \\
*\end{array}$ & $\begin{array}{c}* \\
\mathrm{X} \\
*\end{array}$ & $\begin{array}{l}\mathrm{X} \\
\mathrm{X} \\
*\end{array}$ & $\begin{array}{l}\mathrm{X} \\
\mathrm{X} \\
*\end{array}$ \\
\hline
\end{tabular}

* Fuente: Elaboración propia a partir de los vaciados del PNECU

* Leyenda: "X" el indicador ha sido tratado, "*” el indicador no ha sido tratado, "D" describe, "V" valora

(Dochy, 1991), (De Miguel, 1993). Para ello se establecieron dos tipos de indicadores, unos inmediatos; que aluden a las calificaciones que obtienen los alumnos a lo largo de su período universitario, y otros diferidos, que recogen información acerca de los licenciados en relación con el mercado laboral.

El análisis de los resultados muestra una desorganización de gran parte de los datos y una acusada tendencia descriptiva, pues las facultades ante indicadores tan objetivos como las tasas o medias relacionadas con el rendimiento, no presentan unos análisis y unas valoraciones adecuadas, motivado seguramente por considerar que los datos dan información por sí solos. En próxi- mas convocatorias, es de esperar que este apartado mejore notablemente, pues estos indicadores son valiosos barómetros de la salud de las universidades, aunque sus resultados deben ser examinados teniendo en cuenta los objetivos de la titulación y las expectativas de los alumnos. (R. AlEmany, 2000). En cuanto a los indicadores de graduación sólo Deusto y Granada responden adecuadamente a éstos, aunque simplemente describen. Como última dimensión destaca la valoración y el análisis de los resultados que tienen en cuenta la realidad de los titulados. Este apartado ha sido tratado por Navarra, y sobre todo por la Facultad de Granada, tanto de manera descriptiva como valorativa, debido al uso que este informe hace de los datos cualitativos. 
El estudiante es otra dimensión que toda evaluación universitaria debe tener en cuenta (Cuadro 5), no obstante, cuando el PNECU hace referencia al alumnado no estudia la percepción subjetiva que este tiene sobre la realidad universitaria en la que se inserta (elemento que sí estudia la evaluación de Granada), sino que opta por otras variables relacionadas con la calidad del centro; la existencia de números clausus; todas ellas vinculadas de forma más "directa" con la calidad.

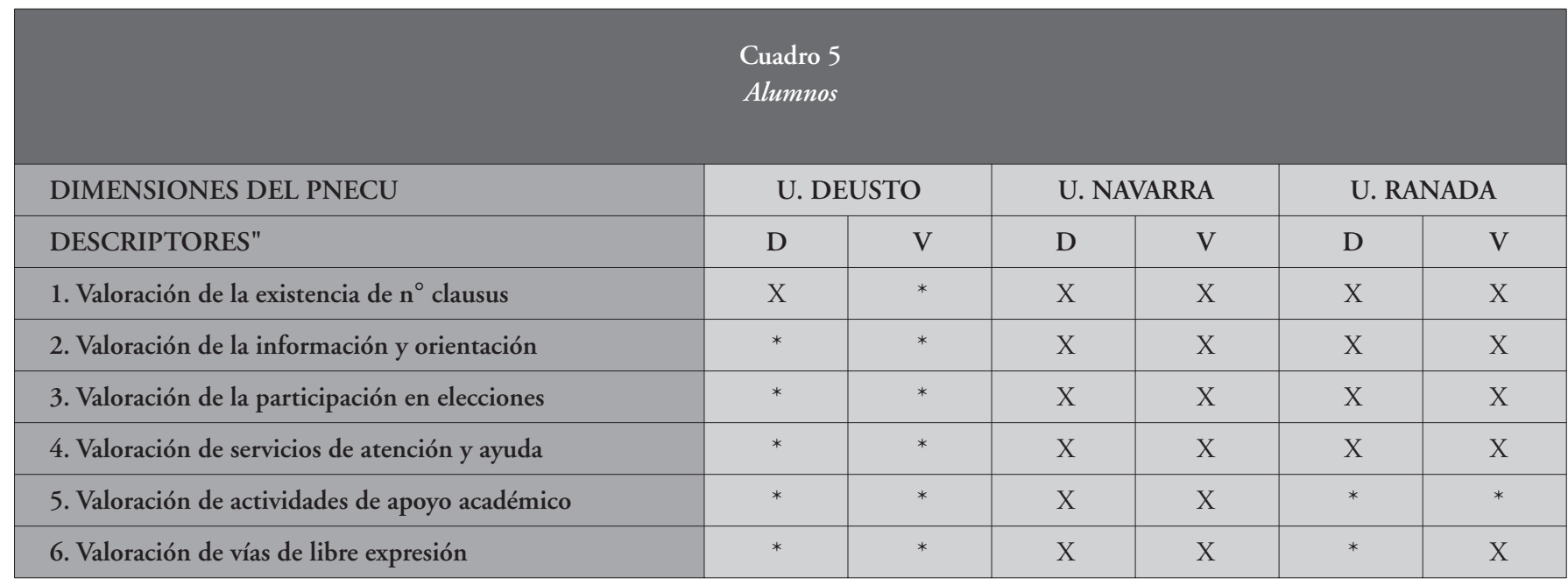

* Fuente: Elaboración propia a partir de los vaciados del PNECU

* Leyenda: "X" el indicador ha sido tratado, "*” el indicador no ha sido tratado, "D" describe, "V" valora

La Facultad de Deusto es la única que no presenta un apartado concreto para la autoevaluación del alumnado, o mejor dicho, la encuesta elaborada para este grupo no abordaba los indicadores recomendados por el Plan. Sin embargo, éste es uno de los puntos que mejor trata Navarra, ya que sí realiza una valoración punto por punto de los indicadores propuestos por la Guía de Evaluación, siendo la información aportada muy cuantitativa, lo que respalda las valoraciones realizadas por el comité. Granada, dedica uno de sus bloques al estudio del alumnado, gracias a lo cual trata casi todos los indicadores propuestos, siendo su rasgo característico la originalidad. Valora aspectos tan novedosos como: el perfil de los alumnos que se han matriculado por primera vez, los hábitos y las formas de estudio o la satisfacción de los mismos.

Otro de los componentes de la comunidad universitaria son los profesores y el personal de administración y servicios (RR.HH.), pilares básicos para el buen funcionamiento de la gestión de la calidad. En lo que respecta a este apartado, sólo la Facultad de Navarra sigue la Guía elaborada por el Consejo de Universidades, destacando sobre todo las evaluaciones de algunos indicadores: como la asignación de profesores y el aprovechamiento de la calidad docente. Los otros dos informes presentan una autoevaluación más "libre", abordando este apartado de manera distinta. Por ejemplo, Deusto recurre a las evaluaciones periódicas que sobre los profesores y las asignaturas realizan los alumnos, fundamentando así su valoración sobre la idoneidad de la plantilla. Granada aporta una serie de indicadores acordes con su filosofía evaluativa y realiza un análisis del currículum, el cum- plimiento de tareas docentes y condiciones de trabajo, la autoevaluación del profesor o la satisfacción con su empleo. Por otro lado, en referencia al PAS, ninguno de los estudios autoevaluativos responde a los indicadores tal y como aconseja el PNECU, sólo Granada presenta algunas alusiones al tema que habían surgido en los grupos de discusión y en las entrevistas en profundidad realizadas a miembros de su plantilla, aunque los temas tratados no estaban muy relacionados con los de la Guía de Evaluación.

Para que un proceso de evaluación sea completo, tanto las instalaciones y las infraestructuras de los centros, como los recursos que éstos poseen, deben ser valorados por los comités de autoevaluación (Cuadro 6). Deusto presenta mayores deficiencias en este punto, pese a dedicarle un pequeño epígrafe a los recursos materiales y realizar una valoración general de las instalaciones, destacando sus diferencias comparativas respecto a las universidades públicas. Sorprende que pese a que la licenciatura de Navarra responda de manera adecuada a las valoraciones sobre las infraestructuras (exceptuando el análisis de la evaluación presupuestaria, que no analizan ninguna de las tres), omita lo referido al estudio concreto de la biblioteca y sólo ofrezca alguna referencia al respecto. En cuanto a Granada, enriquece sus valoraciones con datos procedentes del estudio realizado en 1992, destacando sobre todo la evaluación que realiza de la biblioteca e introduciendo al usuario como evaluador de los servicios que recibe. Un aspecto que ha sido tratado por las tres facultades es la valoración de las instalaciones y las carencias de las mismas, por ello han sido reflejadas en las propuestas de mejora. 


\section{Cuadro 6}

Instalaciones y recursos

\begin{tabular}{|c|c|c|c|c|c|c|}
\hline DIMENSIONES DEL PNECU & \multicolumn{2}{|c|}{ U. DEUSTO } & \multicolumn{2}{|c|}{ U. NAVARRA } & \multicolumn{2}{|c|}{ U. GRANADA } \\
\hline DESCRIPTORES* & $\mathrm{D}$ & $\mathrm{V}$ & $\mathrm{D}$ & $\mathrm{V}$ & $\mathrm{D}$ & $\mathrm{V}$ \\
\hline 1. 1 Valoración de recursos didácticos & * & * & $\mathrm{x}$ & $\mathrm{x}$ & $\mathrm{x}$ & $\mathrm{x}$ \\
\hline 1.2 Valoración de instalaciones & $\mathrm{x}$ & $\mathrm{x}$ & $\mathrm{x}$ & $\mathrm{x}$ & $\mathrm{x}$ & $\mathrm{x}$ \\
\hline 1. 3 Valoración de dotaciones complementarias & * & * & $\mathrm{x}$ & $\mathrm{x}$ & * & * \\
\hline 1. 4 Valoración de carencias relevantes & $\mathrm{x}$ & $\mathrm{x}$ & $\mathrm{x}$ & $\mathrm{x}$ & $\mathrm{x}$ & $\mathrm{x}$ \\
\hline 1. 5 Valoración de espacios y condiciones ambientales & * & * & * & $\mathrm{x}$ & $\mathrm{x}$ & $\mathrm{x}$ \\
\hline 1. 6 Valoración de recursos para docencia e investigación & * & * & $\mathrm{x}$ & $\mathrm{x}$ & $\mathrm{x}$ & * \\
\hline 1. 7 Análisis de la evolución presupuestaria (últimos cinco años) & * & * & $*$ & $*$ & * & * \\
\hline \multicolumn{7}{|l|}{ 2. Biblioteca } \\
\hline 2. 1 Capacidad & * & * & $*$ & * & $\mathrm{x}$ & * \\
\hline 2. 2 Condiciones físicas y funcionalidad & * & * & * & $\mathrm{x}$ & * & * \\
\hline 2. 3 Equipamiento técnico & * & * & $*$ & $*$ & $\mathrm{x}$ & * \\
\hline 2. 4 Recursos humanos & * & * & $*$ & $*$ & $\mathrm{x}$ & $\mathrm{x}$ \\
\hline 2. 5 Adecuación de fondos & * & * & $*$ & $\mathrm{x}$ & * & * \\
\hline 2. 6 Tiempo de demanda-disponibilidad & * & * & * & * & $\mathrm{x}$ & * \\
\hline 2. 7 Sisiemas de acceso y consulta & * & * & * & * & * & * \\
\hline 2. 8 Horario del servicio & * & * & * & * & $\mathrm{X}$ & $\mathrm{X}$ \\
\hline 2. 9 Volumen de consulta y préstamo del profesorado & * & * & * & * & * & * \\
\hline 2. 10 Volumen de consulta y préstamo del alumnado & * & * & * & * & $\mathrm{X}$ & $\mathrm{X}$ \\
\hline
\end{tabular}

* Fuente: Elaboración propia a partir de los vaciados del PNECU.

* Leyenda: "X" el indicador ha sido tratado, "*” el indicador no ha sido tratado", "D" describe, "V"

valorar.

Para conocer de manera más apropiada la situación de las titulaciones han de valorarse las relaciones externas que mantienen los centros con las instituciones públicas y privadas. Pese a la gran importancia de ello en el mundo universitario, la Facultad del País Vasco, no se ha detenido demasiado en este apartado, valorando sólo las relaciones con organizaciones empresariales y la situación de la titulación a nivel nacional e internacional. Navarra tiende a valorar más que a describir con reflexiones bastante satisfactorias, aunque el análisis podía haber sido más enriquecedor si se hubieran presentado algunos datos cuantitativos que corroborasen los juicios de valor. La titulación granadina, destaca por la descripción y la valoración que hace en relación a su entorno más inmediato, no habiendo tratado indicadores más externos a la titulación, como sus relaciones con universidades extranjeras o la participación en programas europeos.

Antes de pasar a tratar el tema de la investigación, algunas de las facultades hicieron una reflexión sobre el proceso de evaluación de la enseñanza que habían llevado a cabo, es decir, el procedimiento de trabajo seguido, los instrumentos e informaciones utilizadas, o el funcionamiento propio del comité. Navarra presenta un epígrafe dedicado a este apartado, que le ha servido para concienciarse de los problemas y plantear soluciones para futuras mejoras. Deusto aunque no dedica un apartado concreto a este análisis, si ha detectado puntos fuertes y débiles relacionados con el mismo. Por último, Granada describe como se realizó el proceso evaluativo, pero no presenta una valoración profunda del mismo que resalte las debilidades encontradas.

Como recuerda Ortega y GaSSeT en su libro Misión de la Universidad (1976), uno de los pilares básicos de esta institución es la investigación, la legendaria Academia aparte de transmitir conocimientos debe producirlos para obtener un desarrollo que proporcione mayor bienestar a la sociedad. Debido a la especial importancia que se le ha otorgado a la investigación universitaria en los últimos años y al aumento cuantitativo de la producción científica, el PNECU ha recomendado una evaluación profunda de la misma. Al igual que en la enseñanza, se establecen unas directrices que quedan recogidas en siete dimensiones y que han sido estudiadas de diversas formas por las facultades objeto de estudio. A pesar de esto, algunos autores (V. Tricio, A. Vol, R. VIlora, 2000) y Comunidades Autónomas (Andalucía y Cataluña), consideran que se olvidan aspectos relevantes tales como las estancias de investigación, conferencias y seminarios, cursos y jornadas, la participación en tribunales, pruebas y congresos. La Comunidad Andaluza, además, considera que no se le presta la suficiente atención a los estudios de tercer ciclo.

Deusto, al tratar la estructura de la Facultad, dedica dos apartados a la investigación. En el primero, hace referencia a las lí- 
neas, relaciones y dificultades que existen en la Facultad, y en el segundo, recoge datos sobre la producción científica del Departamento. Navarra, pese a señalar la evaluación de la investigación como uno de sus grandes objetivos, no presenta unos resultados completos, a pesar de recoger todas las tablas recomendadas por la Guía de Evaluación, no ofrece suficiente información. A pesar de ello, elabora una serie de puntos fuertes y débiles que caracterizan la actividad investigadora de la unidad evaluada. Por otro lado, Granada estudia este apartado de forma diferente a lo establecido por la Guía. No tiene un bloque específico que aborde esta cuestión, aunque cuando evalúa al profesorado ofrece datos de su actividad investigadora (siendo la unidad evaluada el profesorado y no el Departamento).

El contexto, al igual que en la enseñanza, es determinante en la actividad investigadora, quizás sea por esto por lo que todos los indicadores señalados en la Guía son analizados por los comités (Cuadro 7). El problema que plantea este apartado es la falta de evidencias en las valoraciones, sólo Granada -en las dimensiones que analiza- a través de las entrevistas y de los grupos de discusión llega a conclusiones que tienen respaldo empírico. Como indicador importante, resalta la relación entre docencia e investigación, donde tanto Deusto como Navarra hacen una descripción y valoración de las dificultades encontradas en sus centros para compatibilizar estas dos tareas. En Deusto "la docencia académica dificulta una estrategia en el rendimiento por parte de los profesores investigadores", y en Navarra, "el Plan de estudios y la práctica docente imperante en las licenciaturas impartidas por el Departamento, no facilita la correlación docencialinvestigación". La Facultad andaluza por su parte, no estudia esta dimensión como explica la Guía, sino que va más allá, pues intenta conocer la relación existente entre la materia que imparte cada profesor y la actividad en la que investiga, esto permite tanto un enriquecimiento de las clases, como de la propia investigación.

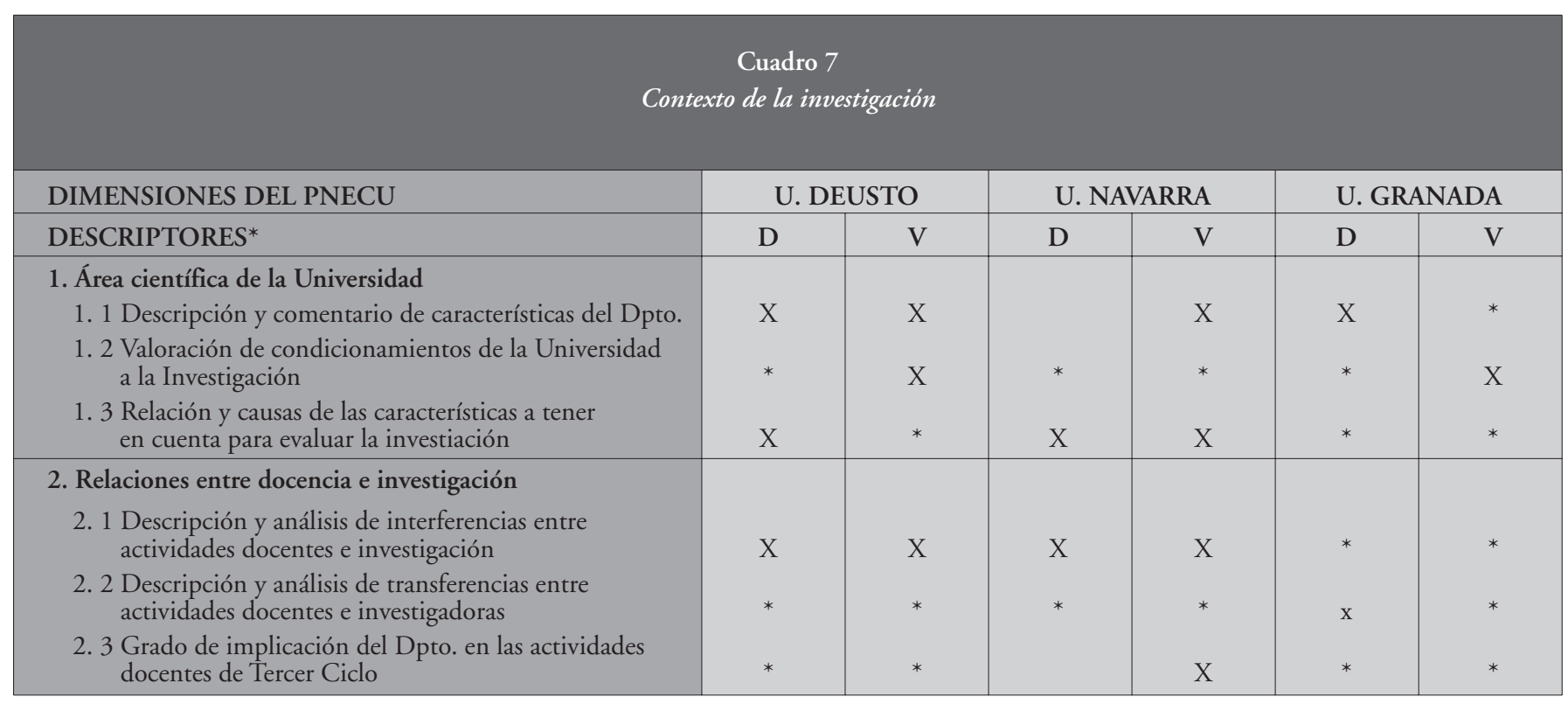

* Fuente: elaboración propia a partir de los vaciados del PNECU

* Leyenda: "X" el indicador ha sido tratado, "*" el indicador no ha sido tratado, "D" descibe, "V" valora

Los departamentos deben definir los planes de investigación que han de estar basados en unos objetivos generales, estos deben ser conocidos por todos sus miembros, como criterio básico para conseguir una investigación eficaz y actualizada ( $R$. ZORRILLA, 2000). A pesar de ello, las facultades del norte reconocen la inexistencia de planes de actuación que contemplen la investigación, aunque las causas sean distintas, unas líneas del informe de Navarra, pueden servir para ilustrar la realidad de las dos facultades "ausencia de un plan conjunto y estratégico en el Departamento orientado al estímulo de la actividad investigadora". Esto también sucede en Granada, como lo demuestra la encuesta realizada a profesores donde se menciona mayoritariamente que los objetivos son difusos (Cuadro 8). El PNECU no se conforma sólo con conocer las líneas principales de investigación, sino que propone una comparación entre los diferentes grupos a nivel nacional, europeo y regional. 
Cuadro 8

Objetivos de la investigación

\begin{tabular}{|c|c|c|c|c|c|c|}
\hline DIMENSIONES DEL PNECU & \multicolumn{2}{|c|}{ U. DEUSTO } & \multicolumn{2}{|c|}{ U. NAVARRA } & \multicolumn{2}{|c|}{ U. GRANADA } \\
\hline DESCRIPTORES* & $\mathrm{D}$ & V & $\mathrm{D}$ & $\mathrm{V}$ & $\mathrm{D}$ & V \\
\hline $\begin{array}{l}\text { 1. Descripción de planes de actuación de actividades } \mathrm{I}+\mathrm{D} \\
\text { en indicación de donde se recogen }\end{array}$ & $\mathrm{X}$ & $\mathrm{X}$ & $\mathrm{X}$ & $\mathrm{X}$ & $\mathrm{X}$ & * \\
\hline 2. ¿Quién y cómo definen planes y objetivos de investigación? & $\mathrm{X}$ & $*$ & $\mathrm{X}$ & $\mathrm{X}$ & $\mathrm{X}$ & * \\
\hline 3. ¿Son conocidos los planes por todos los miembros del Dpto.? & * & $*$ & * & * & $*$ & * \\
\hline $\begin{array}{l}\text { 4. Valoración del peso de la política de investigación a nivel } \\
\text { UE, Nacional y Comunitario en el Dpto. }\end{array}$ & * & $\mathrm{X}$ & $\mathrm{X}$ & * & $*$ & * \\
\hline $\begin{array}{l}\text { 5. Valoración de la investigación del Dpto. dentro de los } \\
\text { objetivos de la política científica } \\
\text { 5. } 1 \text { Nacional } \\
\text { 5. } 2 \text { Europea } \\
\text { 5. } 3 \text { Regional }\end{array}$ & $\begin{array}{l}X \\
X \\
X \\
X\end{array}$ & $\begin{array}{l}X \\
X \\
X \\
X\end{array}$ & $\begin{array}{l}\mathrm{X} \\
* \\
* \\
*\end{array}$ & $\begin{array}{l}* \\
* \\
* \\
*\end{array}$ & $\begin{array}{l}* \\
* \\
* \\
*\end{array}$ & $\begin{array}{l}* \\
* \\
* \\
*\end{array}$ \\
\hline 6. Existencia de planificaón en temas de investigación & $*$ & $*$ & $*$ & $\mathrm{X}$ & $\mathrm{X}$ & * \\
\hline $\begin{array}{l}\text { 7. Inserción de la actividad I+D del Dpto. en los planes } \\
\text { de la Universidad }\end{array}$ & $*$ & $*$ & $*$ & * & $*$ & * \\
\hline
\end{tabular}

*Fuente: elaboración propia a partir de los vaciados del PNECU

* Leyenda: "X" el indicador ha sido tratado, "*" el indicador no ha sido tratado, "D" describe, "V" Valora

La titulación de Deusto, a pesar de no tener una actividad investigadora intensa, sí muestra interés en conocer su posición con respecto a las relaciones que mantienen sus investigadores, tanto a nivel nacional como europeo. Para este comité, el ser una institución de carácter privado dificulta la relación con los organismos públicos, así reconoce que "la existencia de cierta lejanía con las instituciones de las instancias públicas, tanto del Gobierno Autónomo Vasco, como del Central Español'. Las otras dos licenciaturas no han tenido en cuenta estas dimensiones, aunque
Granada analiza las publicaciones internacionales que tienen los profesores del Departamento y la asistencia a distintos congresos.

Muy relacionado con los objetivos se encuentran los recursos, siendo esta una de las mayores deficiencias con las que cuentan las universidades españolas en materia investigadora pese al esfuerzo realizado en los últimos años. Quizá sea por ello y por la facilidad de acceso a la información, por lo que este apartado es tratado por todos los informes (Cuadro 9 ).

\begin{tabular}{|c|c|c|c|c|c|c|}
\hline DIMENSIONES DEL PNECU & \multicolumn{2}{|c|}{ U. DEUSTO } & \multicolumn{2}{|c|}{ U. NAVARRA } & \multicolumn{2}{|c|}{ U. RANADA } \\
\hline DESCRIPTORES" & $\mathrm{D}$ & V & $\mathrm{D}$ & $\mathrm{V}$ & $\mathrm{D}$ & $\mathrm{V}$ \\
\hline 1. Valoración de datos sobre RR.HH & $\mathrm{X}$ & $\mathrm{X}$ & $\mathrm{X}$ & * & $\mathrm{X}$ & $\mathrm{X}$ \\
\hline 2. Valoración de recursos ecónomicos & * & $\mathrm{X}$ & $\mathrm{X}$ & * & $*$ & $\mathrm{X}$ \\
\hline 3. Valoración de recursos materiales & * & $\mathrm{X}$ & $\mathrm{X}$ & $\mathrm{X}$ & * & * \\
\hline
\end{tabular}

* Fuente: Elaboración propia a partir de los vaciados del PNECU .

* Leyenda: "X" el indicador ha sido tratado, "*” el indicador no ha sido tratado, "D" describe, "V" valora

La manera en que las facultades han tratado estas dimensiones es desigual. La Facultad andaluza, pese a no seguir lo dictado por el PNECU, ofrece una serie de valoraciones recogidas por medio de la opinión de los profesores, que son analizadas en el apartado dedicado al Rectorado. Como nota característica, destaca que existe un descontento general respecto a los recursos, se afirma que "tanto la dotación presupuestaria de la Facultad como el apoyo a la investigación es insuficiente". Las otras dos titulaciones 
presentan una tendencia diferente, mientras que Navarra ofrece todas las tablas propuestas y una descripción cuantitativa del tema acompañada por muy pocas valoraciones, Deusto hace todo lo contrario o, lo que es lo mismo, no presenta datos descriptivos relevantes, pero sí recoge juicios de valor que deben ser considerados, destacando sobre todo la siguiente reflexión: "la casi totalidad de los recursos están concentrados en atender las demandas ordinarias del proceso formativo de los alumnos... lo que impide liberar recursos para acometer proyectos de cierta entidad".

Tras haber analizado el contexto en el que se encuentran los grupos de investigación, así como los objetivos propuestos y los recursos de los que disponen, el siguiente apartado a valorar es la estructura de los equipos de investigación y las relaciones que establecen en su actividad cotidiana, ya que no hay que olvidar la importancia que tiene la interacción tanto entre los grupos de investigación, como entre los miembros de dichos grupos, para poder llevar a cabo una investigación más completa (Cuadro 10).
En el análisis de este epígrafe hay que diferenciar las relaciones que se producen dentro de la institución y las relaciones con otras áreas de conocimiento; características éstas, que llevan a consolidar líneas de investigación cuyas aportaciones a los departamentos y universidades deben ser valoradas. Tampoco se deben olvidar los problemas, sobre todo burocráticos y administrativos que suelen acompañar a la tarea investigadora. Con el objetivo de integrar la labor científica en la Universidad, se deben analizar las relaciones con otras instituciones, diferenciándose según el tipo y el carácter -público o privado- a la que se pertenezca, se debe también valorar el seguimiento prestado por éstas y el grado de dificultad en las relaciones con instituciones públicas. En cuanto a las relaciones de la investigación con el ámbito privado, se debe medir su grado de satisfacción, exigencias, y el tipo de necesidades detectadas, ya que esto puede ayudar a concienciar a los equipos de investigación de las dificultades existentes en este ámbito.

Cuadro 10

Estructura de la investigación

\begin{tabular}{|c|c|c|c|c|c|c|}
\hline DIMENSIONES DEL PNECU & \multicolumn{2}{|c|}{ U. DEUSTO } & \multicolumn{2}{|c|}{ U. NAVARRA } & \multicolumn{2}{|c|}{ U. RANADA } \\
\hline DESCRIPTORES* & $\mathrm{D}$ & V & $\mathrm{D}$ & V & $\mathrm{D}$ & V \\
\hline $\begin{array}{l}\text { 1. Relación dentro de la institución } \\
\text { 1. } 1 \text { Existencia de líneas de investigación estables } \\
\text { 1. } 2 \text { Valoración de creación y continuidad de grupos } \\
\text { de investigación } \\
\text { 1. } 3 \text { Existencia de problemas que impidan la creación } \\
\text { de grupos de investigación } \\
\text { 1. } 4 \text { Análisis de relaciones existentes entre distintas } \\
\text { áreas de conocimiento } \\
\text { 1. } 5 \text { Análisis de relaciones de investigación con otras disciplinas } \\
\text { 1. } 6 \text { Valoración del grado de saturación de investigadores } \\
\text { con tareas administrativas } \\
\text { 1. } 7 \text { Valoración de las relaciones con los servicios } \\
\text { de gestión de la investigación } \\
\text { 1. } 8 \text { Valoración del conocimiento de la Universidad } \\
\text { sobre la oferta científica y tecnológica del Dpto }\end{array}$ & $\begin{array}{l}\mathrm{X} \\
* \\
* \\
* \\
* \\
*\end{array}$ & $\begin{array}{l}* \\
*\end{array}$ & $\begin{array}{c}\mathrm{X} \\
*\end{array}$ & $\begin{array}{l}\mathrm{X} \\
\mathrm{X} \\
\mathrm{X} \\
\mathrm{X} \\
* \\
* \\
* \\
\text { * } \\
\mathrm{X}\end{array}$ & $\begin{array}{l}* \\
* \\
* \\
\text { X } \\
* \\
* \\
*\end{array}$ & $\begin{array}{l}* \\
*\end{array}$ \\
\hline $\begin{array}{l}\text { 2. Relaciones con otras instituciones } \\
\text { 2. } 1 \text { Análisis de tipologías de instituciones relacionadas } \\
\text { con investigación del Dpto } \\
\text { 2. } 2 \text { Valoración de la dificultad de establecer relaciones } \\
\text { con diferentes instituciones públicas } \\
\text { 2. } 3 \text { Valoración del seguimiento del resultado por las } \\
\text { instituciones públicas } \\
\text { 2. } 4 \text { Existencia de relaciones de investigación } \\
\text { condicionadas con otras instituciones } \\
\text { 2. } 5 \text { Origen del establecimiento de relaciones } \\
\text { con otras instituciones } \\
\text { 2. } 6 \text { Valoración de las relaciones de colaboración con empresa } \\
\text { 2.6. } 1 \text { Grado de satisfacción } \\
\text { 2. 6. } 2 \text { Características específicas } \\
\text { 2. 6. } 3 \text { Nivel de exigencia } \\
\text { 2. } 6.4 \text { Tipo de necesidades detectadas o resueltas } \\
\text { 2. } 7 \text { Análisis de la proporción de relaciones } \\
\text { con instituciones públicas o privadas } \\
\text { 2. } 8 \text { Causa de las relaciones con instituciones públicas o privadas }\end{array}$ & $\begin{array}{l}* \\
* \\
* \\
* \\
* \\
*\end{array}$ & $\begin{array}{l}\mathrm{X} \\
* \\
* \\
\text { X }\end{array}$ & $\begin{array}{l}* \\
* \\
* \\
*\end{array}$ & $\begin{array}{l}\mathrm{X} \\
* \\
* \\
* \\
\\
* \\
*\end{array}$ & $\begin{array}{l}\mathrm{X} \\
* \\
* \\
* \\
* \\
* \\
\mathrm{X} \\
\mathrm{X} \\
* \\
* \\
\mathrm{X} \\
* \\
*\end{array}$ & $\begin{array}{l}* \\
* \\
* \\
*\end{array}$ \\
\hline
\end{tabular}

* Fuente: elaboración propia a partir de los vaciados del PNECU

* Leyenda: "X" el indicador ha sido tratado, "*” el indicador no ha sido tratado, "D" describe, "V" Valora 
En general, los indicadores destinados a evaluar las relaciones dentro de la institución han despertado un gran interés, sobre todo en las facultades del norte. Éstas analizan las líneas de investigación existentes, que no siempre se corresponden con grupos de investigación estables. Ambos informes destacan los diversos problemas que obstaculizan la tarea científica y recogen valoraciones que responden a algunos de los indicadores propuestos. Deusto vuelve a señalar las dificultades económicas como la causa principal que le impide realizar más y mejores investigaciones, recordando su carácter privado, que hace que sus mayores esfuerzos vayan destinados a la docencia. El caso de Navarra es también un ejemplo de lo expuesto hasta ahora, ya que los problemas con los que se encuentran son propios de la licenciatura, y van más allá de la propia investigación, presentando causas como la juventud del departamento, la tradición de pequeños grupos de investigación donde predomina el interés formativo, las conveniencias estratégicas, la dificultad para estimar la actividad investigadora, etc.

El segundo bloque de indicadores hace referencia a las relaciones que mantienen los equipos de investigación con otras instituciones. Los resultados obtenidos en el análisis de estas dimensiones son deficientes y escasos, y sólo la relación de las instituciones con los departamentos y la relevancia en foros nacionales e internacionales de los profesores son analizados por más de un informe. Lo más destacado de este epígrafe es que no se valoren adecuadamente las relaciones de los grupos de investigación con las empresas de la zona, motivado seguramente por el poco contacto existente entre las mismas. Granada, aparte de estudiar algunos de los indicadores que aparecen en la Guía de evaluación, como las relaciones entre áreas de conocimiento, las investigaciones terminadas o en marcha y los contratos de investigación, incluye otros aspectos novedosos y muy relacionados con esta dimensión, como las relaciones de poder y las presiones entre grupos dentro del Departamento.

Una vez evaluada la situación de la investigación, se procede a conocer los resultados de la producción científica o, lo que es lo mismo, se juzga si los resultados que se obtienen están acorde con los medios disponibles, aunque hay que recordar que está dimensión debería ir acompañada de unos criterios de calidad, para no sólo cuantificar, sino también cualificar (HOUSE y YEAGER, 1979). La facultad vasca y la de Navarra ofrecen una serie de tablas que se aproximan a lo exigido, presentando una evaluación de los resultados de la investigación, tanto en lo que se refiere a tesis doctorales, como asistencia a congresos y publicaciones. Destaca sobre todo la titulación de Deusto, que no se limita sólo a ofrecer cifras, sino que éstas se complementan con comentarios y valoraciones que explican el porqué de su productividad, mientras que Navarra sólo presenta los datos exigidos, valorando nada más que el indicador referido a tesis doctorales.
Por otro lado, Granada ofrece un gráfico donde se muestran los porcentajes referidos a la producción científica de sus profesores -publicación de libros, artículos, presentación de ponencias y conferencias- los cuales son valorados. El que las valoraciones estén basadas en evidencias en lo que a la producción científica se refiere tiene una gran importancia, pues un buen indicador para financiar la actividad científica de los distintos departamentos puede ser la productividad investigadora de los grupos de investigación, ya que la financiación de las instituciones puede establecerse en función de los éxitos (DOBSON, SHARMA, HAYDON, 1998).

Los indicadores referidos al rendimiento (Cuadro 11) deben responder al empleo de los recursos, a la eficiencia y eficacia de los resultados, para así rendir cuentas a la sociedad (J. SizER, 1991). Este indicador tradicionalmente tratado de manera objetiva debe estar acompañado de otros que hagan referencia a la calidad, ya que su último fin es acercarse mejor a las actividades que se realizan dentro de la institución (FrankManN y MufFo, 1998). No hay que olvidar que el dato interesa, pero lo más importante es la finalidad, que no es otra que mejorar la calidad de la investigación. Las titulaciones analizadas han respondido a la mayoría de estos indicadores, pero vuelven a repetirse las tendencias aparecidas en otros apartados, es decir, Granada aporta nuevas dimensiones para este estudio (el carácter individual o grupal de la investigación o la participación del alumnado entre otros) y concluye afirmando que "en lineas generales hay que destacar el considerable volumen de las actividades realizadas por el profesorado de la facultad en el último año". Navarra presenta los cuadros exigidos por la Guía de Evaluación, pero de nuevo no establece valoraciones profundas. Deusto, presenta tablas y comentarios, e incluso es la única Facultad que responde a los indicadores de éxito y concentración.

En lo que a la evolución de la investigación se refiere, Granada vuelve a responder con originalidad, incluyendo un indicador que analiza las horas que cada profesor dedica a su actividad investigadora, según su categoría profesional y comparando los resultados con los de la evaluación llevada a cabo en 1992. El único indicador que ha sido tratado por todas las facultades es el que hace referencia a la descripción de factores decisivos para la investigación, y que las facultades consideran que se encuentran dentro de la propia institución. Como último aspecto, sorprende que no se evalúen las causas que influyen de manera decisiva en la evolución de la investigación. Igualmente, y a pesar de la importancia que tiene el fenómeno de la calidad en la investigación (IGLESIAS DE UsSEL, 2000), ningún comité ha dedicado un apartado concreto para el estudio del tema, como se desprende del análisis del último indicador. 


\begin{tabular}{|c|c|c|c|c|c|c|}
\hline DIMENSIONES DEL PNECU & & & & & $\mathrm{U}$. & \\
\hline DESCRIPTORES* & $\mathrm{D}$ & V & $\mathrm{D}$ & V & $\mathrm{D}$ & $\mathrm{V}$ \\
\hline 2. Éxito & $\mathrm{X}$ & $\mathrm{X}$ & $*$ & $*$ & * & $*$ \\
\hline 3. Productividad & * & $*$ & $\mathrm{X}$ & $\mathrm{X}$ & $\mathrm{X}$ & $\mathrm{X}$ \\
\hline 4. Concentración & $\mathrm{X}$ & $\mathrm{X}$ & $*$ & $*$ & * & * \\
\hline $\begin{array}{l}\text { 5. Evolución } \\
\text { 5. } 1 \text { Análisis y valoración de indicadores de evolución } \\
\text { 5. } 1.1 \text { RR.HH } \\
\text { 5. 1. } 2 \text { Obtención de becas y proyectos de investigación } \\
\text { 5. } 1.3 \text { Firma de contratos } \\
\text { 5. } 2 \text { Análisis de resultados } \\
\text { 5. } 3 \text { Análisis de causas de la evolución } \\
\text { 5. } 4 \text { Descripción de factores } \\
\text { 5. } 4.1 \text { En la institución } \\
\text { 5. } 4.2 \text { En ámbito nacional } \\
\text { 5. } 4.3 \text { En ámbito internacional }\end{array}$ & $\begin{array}{c}* \\
\mathrm{X} \\
* \\
\mathrm{X} \\
*\end{array}$ & $\begin{array}{c}* \\
\mathrm{X} \\
* \\
\mathrm{X} \\
*\end{array}$ & $\begin{array}{l}\mathrm{X} \\
\mathrm{X} \\
\mathrm{X} \\
\mathrm{X} \\
\mathrm{X} \\
\\
\mathrm{X} \\
* \\
*\end{array}$ & $\begin{array}{l}* \\
* \\
* \\
* \\
* \\
\text { X } \\
* \\
* \\
\end{array}$ & $\begin{array}{c}\mathrm{X} \\
* \\
\mathrm{X} \\
? \\
* \\
\mathrm{X} \\
* \\
*\end{array}$ & $\begin{array}{l}\mathrm{X} \\
* \\
* \\
\text { ? } \\
\text { * }\end{array}$ \\
\hline $\begin{array}{l}\text { 6. Calidad } \\
\text { 6. } 1 \text { Análisis y valoración de actividades de investigación del Dpto. } \\
\text { 6. 1. } 1 \text { Tipo de becas } \\
\text { 6. 1. } 2 \text { Proyectos } \\
\text { 6. 1. } 3 \text { Contratos } \\
\text { 6. 1. } 4 \text { Resultados de la investigación } \\
\text { 6. } 2 \text { Descripción de criterios para determinar } \\
\text { la calidad de la investigación }\end{array}$ & $\begin{array}{l}\mathrm{X} \\
\mathrm{X} \\
* \\
\mathrm{X} \\
\\
\end{array}$ & $\begin{array}{l}* \\
* \\
* \\
\text { X }\end{array}$ & $\begin{array}{l}X \\
X \\
X \\
X \\
\\
\end{array}$ & $\begin{array}{l}* \\
\mathrm{X} \\
\mathrm{X} \\
\mathrm{X} \\
*\end{array}$ & $\begin{array}{l}* \\
\mathrm{X} \\
\mathrm{X} \\
\mathrm{X}\end{array}$ & $\begin{array}{l}* \\
* \\
* \\
* \\
*\end{array}$ \\
\hline
\end{tabular}

* Fuente: elaboración propia a partir de los vaciados del PNECU

* Leyenda: "X" el indicador ha sido tratado, "*” el indicador no ha sido tratado, "D" describe, "V" valora, "?" compara los datos con la evaluación de 1992

Tras comparar los indicadores de investigación en los distintos informes, se observa que todas las facultades podían haber dedicado una mayor atención a este apartado, aunque al seguir el Informe Final de Evaluación, se aprecia que se cumplen las mismas pautas que para el resto de las titulaciones evaluadas. Por todo ello, es conveniente señalar que el seguimiento de la Guía de Evaluación ha sido escaso en esta dimensión, y que existen muchas valoraciones que no están acompañadas de evidencias y muchos datos que no son valorados ni comentados.

El tercer bloque de análisis es el referido a la gestión. Ésta es la dimensión menos tratada por las facultades y universidades que concurrieron a la II convocatoria del PNECU, seguramente motivado por la falta de información de los equipos evaluativos y por la poca homogeneidad que existe en la clasificación de la información requerida. A pesar de ello, en toda evaluación es necesario un sistema de indicadores que refleje el desenvolvimiento real de la institución y que incluya estadísticas relativas a la satisfacción de los clientes, a la eficiencia interna de los proce- sos, y al nivel de innovación y aprendizaje del personal (A. REY GARCÍA, 1988). El sistema que propone la Guía de Evaluación ha sido elaborado a partir del "Modelo Europeo para la Gestión de la Calidad Total", diseñado por la "European Foundation for Quality Management (EFQM), cuyo objetivo es el "análisis de los sistemas de calidad de una unidad que le permite gestionar los recursos a través de los procesos, para conseguir unos determinados resultados, incorporando una dinámica de mejora continua y sistémica”. Según J. GraO, y R. Winter (2000), sus categorías abarcan todos los aspectos de la organización y sirve para identificar las características de lo que debería ser y la realidad, proponiendo planes de mejora medibles y consistentes, es decir, que los planes de acción lleven a resultados.

Si hubiera que clasificar los informes por los datos aportados sobre este tema, Granada ocuparía el primer lugar, seguido de Deusto y Navarra, que apenas hacen mención a la gestión universitaria. Debido a la falta de datos, a la heterogeneidad de los mismos y la libertad que ofrece la Guía de Evaluación para clasi- 
ficar esta información, no se seguirá ningún modelo específico para el análisis de estos indicadores, sino que se explicará lo que ha realizado cada uno de los comités.

El informe presentado por la Facultad de Granada dedica dos bloques a la organización y funcionamiento del centro y el Rectorado. En un primer análisis se observa que se ha evaluado tanto el funcionamiento administrativo de la Facultad, como la relación que ésta tiene con el Rectorado y con otros agentes externos. El estudio se fundamenta en las opiniones tomadas mediante encuestas y grupos de discusión de los profesores y alumnos, aunque para aspectos tan importantes como éstos se recurrió a realizar seis entrevistas semiestructuradas a miembros del P.A.S y cinco a personas integrantes del equipo decanal. Por medio de estas técnicas de investigación se conoció: la organización docente, la gestión de recursos, el funcionamiento de los órganos colegiados y unipersonales, los servicios de biblioteca, secretaría y conserjería, la toma de decisiones y todo lo concerniente a la información (ambiente y relaciones de los servicios administrativos de la Facultad). A diferencia de otros informes, éste se caracteriza por tener siempre presente al usuario, es decir, es una evaluación centrada en la opinión que tienen los demandantes del servicio que reciben. Esto sin duda, es muestra del afianzamiento de la cultura de calidad en este centro en los últimos años.

Cuando el comité de evaluación de Granada estudia la toma de decisiones, lo hace a partir de siete subdimensiones que recogen las opiniones de profesores y alumnos sobre participación, información, responsables, grado de acuerdo, cumplimiento, presiones y motivaciones. Para el análisis de esta dimensión, el informe ha recogido datos cuantitativos y cualitativos, y comprara los resultados obtenidos con los de la evaluación de 1992. Como nota característica de este apartado, destaca una mayor implicación de los alumnos y la percepción de "desgana con una mezcla de desinterés" en la participación de los profesores. Aunque uno de los objetivos de este punto es estudiar la responsabilidad e implicación en la toma de decisiones, esto no es estudiado con la profundidad que se debiera, pues se ha estimado más oportuno profundizar en los grupos de presión que mediatizan las decisiones y que tienen una marcada presencia en la influencia en estos procesos.

En lo que a los departamentos se refiere se han evaluado temas como: la coordinación interna, la división de funciones, composición, cohesión, clima, toma de decisiones, organización y control del profesorado. El análisis de este apartado está fundamentado sobre todo en las valoraciones de los profesores sobre sus actuaciones y funciones. Las opiniones de los docentes son recogidas a partir de un cuestionario donde se les pregunta sobre su grado de acuerdo o desacuerdo en la participación de la vida del departamento, la aceptación de iniciativas por parte del equipo directivo, la toma de decisiones en los órganos correspondien- tes, la participación en actividades organizadas por compañeros o sobre el control de sus funciones. Igual que en el apartado anterior los resultados son comparados con los de la primera evaluación, destacando dos elementos claves para el funcionamiento del departamento, como son la definición de funciones y la participación en actividades. La opinión de los alumnos en este apartado no ha sido suficientemente valorada, aunque sí se recogen algunas reclamaciones que han surgido en los grupos de discusión, estas voces pedían una mayor organización en el funcionamiento de los departamentos. Seguramente la poca importancia dada a los estudiantes en este apartado (M. MiguEL DE, 2000) esté justificada por la falta de motivación y expectativas que éstos muestran ante las escasas posibilidades de cambio de las estructuras académicas.

En el análisis de la gestión universitaria, no se pueden olvidar todos aquellos servicios que presta una Facultad. Así, este informe evalúa la secretaría administrativa y la biblioteca por medio de las valoraciones que de estos servicios hacen los usuarios/clientes. Respecto al primer indicador se miden seis subdimensiones, que tienen el objetivo de valorar el servicio que prestan. Se estudia la eficacia, la información, la disponibilidad, la capacidad administrativa, el personal y los servicios que desarrollan. Este apartado adquiere importancia al ser los usuarios los que opinan sobre las cuestiones del servicio, lo que permite detectar las debilidades que presenta la administración a la hora de responder a las demandas que se le plantean. Con la misma metodología e intención se aborda el tema de la biblioteca, que es un servicio clave de apoyo a las dos funciones que constituyen la razón de ser de la institución universitaria: la investigación o creación de conocimiento y la enseñanza o comunicación de dicho conocimiento (J. ARÉVALO, 2000). Así se miden aspectos como: el horario, la organización, la efectividad, la accesibilidad, la atención y el funcionamiento. Como aspecto negativo hay que resaltar que no aparecen datos que reflejen aspectos tan relevantes como: el número de libros comprados por mes, el presupuesto disponible o la diferencia que existe entre usuarios reales y potenciales. A nivel metodológico se observa que la encuesta suministrada para este apartado no ha cumplido una serie de requisitos (tamaño de la muestra, aleatoriedad...), por lo tanto estos resultados deben interpretarse con cierta cautela y no hacer valoraciones globales de los mismos. Un indicador original que aporta el comité andaluz es el perfil de los usuarios de este servicio, controlando o presentando variables como el sexo, la frecuencia de uso, los usos más frecuentes o las revistas más consultadas.

La información es otro de los elementos claves para el buen funcionamiento de la gestión (así lo recoge la Guía de Evaluación), ésta debe de ser fluida, accesible y clara para que permita la trasparencia en los procesos de organización del servicio y toma de decisiones. Para medir esta dimensión el informe de Granada presenta cinco subdimensiones que tratan de analizar los canales de información, la publicidad de la misma, la pun- 
tualidad, la accesibilidad y los temas. Como en apartados anteriores, se vuelve a preguntar a los profesores y los alumnos sobre la información que tienen sobre cuestiones como: el presupuesto de la Facultad, la elaboración del mismo, las prácticas de empresa, las decisiones del equipo decanal y los acuerdos de la Junta de Facultad.

Este informe también ha tenido presente las relaciones de la Facultad con el Rectorado. Los aspectos valorados están relacionados con la toma de decisiones, el apoyo a las actividades de la Facultad, la dotación presupuestaria asignada, la fluidez de los canales de comunicación, la atención a las iniciativas que se proponen desde este centro y la difusión de información (accesibilidad, canales, destino, actualización, etc.). Como ocurre en apartados anteriores al ser esta una evaluación basada en los clientes/usuarios y sus percepciones, no se presentan datos que apoyen las evidencias, es decir, se le pregunta a los profesores si la partida presupuestaria que reciben del rectorado es suficiente, pero no se expone el presupuesto oficial con el que cuenta la Facultad.

Las facultades de Deusto y Navarra no estudian la gestión como dice el PNECU, sino que se limitan a ofrecer unas pequeñas notas no muy esclarecedoras sobre la gestión de su centros. Deusto dedica el tercer bloque a estudiar el clima corporativo de la Facultad, recogiendo algunos aspectos relacionados con la gestión. Se menciona la gestión académica, el estatus intracorporativo y el clima organizacional. En primer lugar, se establece una descripción de la estructura organizacional tanto de la Facultad como de la Universidad: "los órganos que intervienen en el diseño de la gestión y su puesta en práctica van desde el claustro del profesorado, el Consejo, la Junta y el Decanato de la Facultad, hasta el Consejo de Dirección y el Rectorado". En segundo lugar, se establece la posición que ocupa la Universidad en el contexto universitario de Deusto. Por último, se recogen opiniones de los profesores sobre el clima organizacional del centro, donde se destacan aspectos como la falta de apoyo administrativo, el estilo de dirección de la Facultad, el peso de la burocracia y la participación real en la toma de decisiones del centro. El comité de autoevaluación de Navarra no ha estudiado esta dimensión, al considerar que debido al carácter central de los servicios y a la poca autonomía dotada a las titulaciones para su gestión, es más oportuno estudiar este apartado desde los servicios que presta el Rectorado.

\section{Conclusiones}

Una vez realizado el estudio comparativo de los informes de autoevaluación emitidos por las tres facultades, se presentan los resultados generales de los indicadores que han sido tratados por cada una de ellas en enseñanza e investigación -se omite la gestión por no haber sido estudiada de manera más o menos homogénea por las titulaciones-, teniendo en cuenta el carácter valorativo y descriptivo de los mismos. Los indicadores propuestos por la Guía de Evaluación para el estudio de la enseñanza recogidos en los vaciados de este trabajo son 116 y para la investigación 64. A partir de estos datos, se presentan los porcentajes correspondientes a los indicadores que, han sido utilizados en cada uno de los informes. Los resultados ofrecidos en los gráficos presentados a continuación, son calculados sobre cien y deben ser interpretados por separado, es decir, por un lado la descripción y por otro la valoración que se hace de las dimensiones propuestas, no distinguiéndose si las valoraciones previamente han sido descritas y están basadas en evidencias.

De las tres titulaciones, Navarra es la que más se aproxima al PNECU (Gráfico 1), aunque Granada a pesar de no seguir lo propuesto por el Consejo de Universidades, describe en el caso de la educación el 59\% de los indicadores recomendados. Pero sólo valora el 36\%. Deusto es la que menos ha utilizado estas dimensiones, seguramente motivado por tener un carácter particular, así coincide en los indicadores descriptivos planteados en un $40 \%$ y sólo hace valoraciones del $25 \%$. Navarra, que como ya se comentó sigue lo dictado por la Guía de Evaluación, no analiza todos los indicadores, es decir, describe el 56\% y valora el $60 \%$.

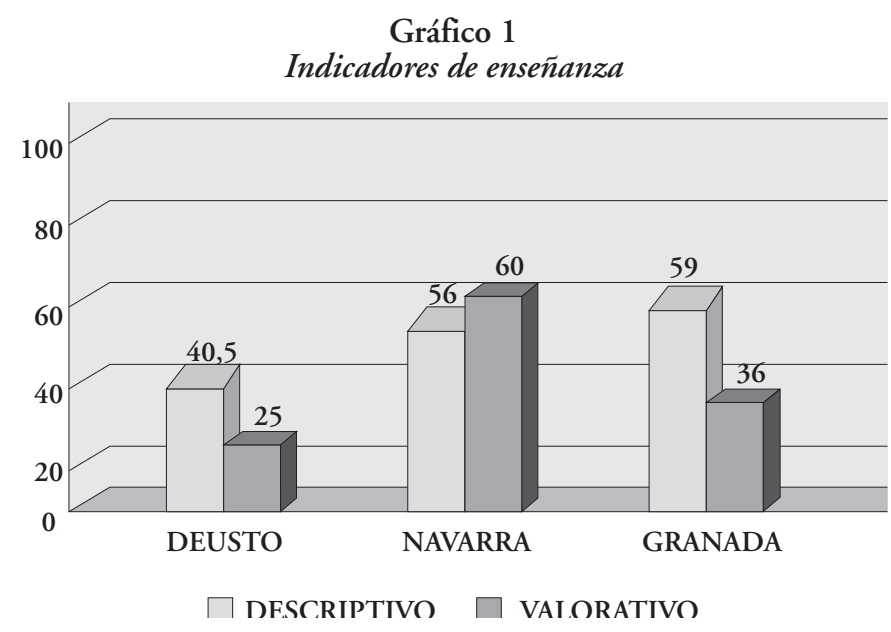

En lo que respecta a la investigación (Gráfico 2) las pautas vuelven a ser las mismas, aunque ahora Granada es la que menos indicadores utiliza de los propuestos por la Guía de Evaluación, es decir, describe un $31 \%$ de las dimensiones y valora el $17 \%$. Deusto por su parte valora más que describe, o lo que es lo mismo, usa aquellos en un $45 \%$ y éstos en un 39\%. Navarra baja sus resultados con respecto a la enseñanza, así describe el 50\% y valora el $33 \%$. 
Gráfico 2

Indicadores de investigación

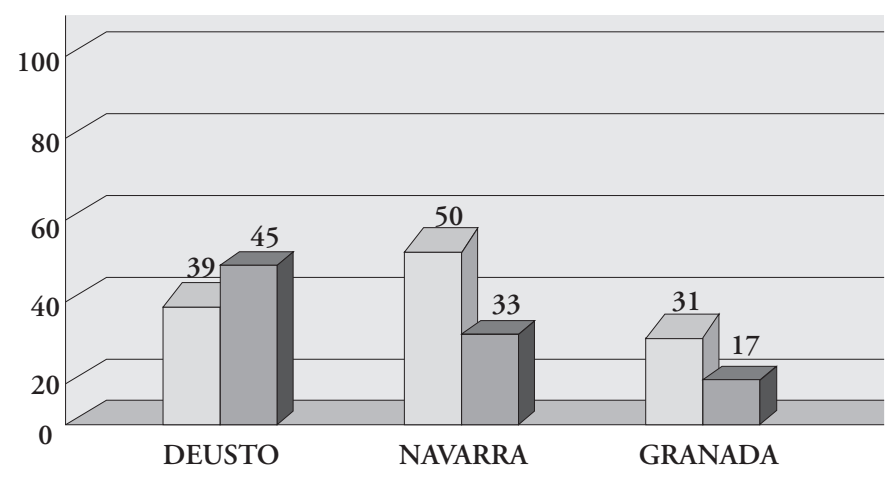

$\square$ DESCRIPTIVO $\square$ VALORATIVO

El análisis cuantitativo de los datos sirve para conocer las diferencias o semejanzas que existen entre las unidades evaluadas y lo recomendado por el Consejo de Universidades, y ayuda a realizar una serie de reflexiones sobre los procesos evaluativos. A pesar de que todas las titulaciones estudiadas han consultado la Guía de Evaluación, todas siguen modelos distintos, lo cual demuestra que el proceso de evaluación no es uniforme, y que existen múltiples vías a través de las cuales se puede conocer la realidad universitaria y conseguir mejorar la calidad -docente, investigadora y de gestión-.

El hecho de que estas facultades realicen un proceso de autoevaluación, es síntoma de una preocupación por la búsqueda de la calidad y una asimilación por parte de la comunidad universitaria de la necesidad de ofrecer una enseñanza más eficaz, eficiente y comprometida con la sociedad. Teniendo en cuenta el contexto en el que se encuentra la universidad española y el futuro que se avecina, la evaluación cada vez adquiere más presencia en los centros, pero ésta no debe ser discontinua, sino que como recomiendan los distintos expertos en la materia debe ser asimilada y llevada a cabo de manera periódica. La continuidad en el tiempo es importante y ayuda a comprobar si las propuestas de mejora se han llevado a cabo y contribuyen a la institucionalización de la cultura evaluativa. Este aspecto, es un punto débil en las evaluaciones analizadas, pues en ninguna de ellas se ha presentado un plan de revisión y seguimiento de las acciones de mejora.

El modelo evaluativo que ha seguido cada una de las titulaciones, debe servir de referencia para mejorar futuras convocatorias. De entre las titulaciones analizadas, destaca la forma en que Granada seleccionó las dimensiones y subdimensiones, ya que obligó a todos los agentes objeto de la evaluación a participar en la misma, o lo que es igual, el concepto de calidad fue definido por todos los miembros que componen la realidad educativa de esta licenciatura. Además, gracias a esta metodología, se ha subsanado uno de los problemas detectados en todas las evaluaciones y en casi todos los procesos evaluativos, las personas estaban informadas de que la Facultad estaba siendo evaluada y no les importaba ser objeto de estudio.

Del análisis de los informes se desprende que debe tenerse presente el diferente contexto en el que surgen las distintas evaluaciones de las titulaciones. Granada destaca por tomar como referencia la evaluación de 1992 que se llevó a cabo en su centro, lo que demuestra que esta facultad tiene una cierta cultura evaluativa. Deusto destaca por su carácter privado, lo que hace que se centre más en la enseñanza, al ser éste un pilar básico para su financiación. Navarra a diferencia de Granada, estimó oportuno seguir lo dictado por la Guía de Evaluación, en vez de crear un propio modelo, aunque no realizó un estudio profundo del contexto, y presentó algunas lagunas en la investigación, omitiendo el estudio de la gestión.

La evaluación de la enseñanza universitaria debe ser entendida como un proceso continuo de reflexión que responda a las preguntas: qué somos, qué tenemos y qué queremos hacer. En líneas generales, las tres titulaciones han respondido a estas preguntas, es decir, han tomado conciencia del lugar que ocupan dentro de su Universidad, han valorado los medios de los que disponen y plantean soluciones y retos de futuro. A pesar del esfuerzo realizado por los comités, falta una mayor visión crítica de la realidad universitaria, muchos de los puntos débiles aparecen justificados de manera difusa o se culpa a terceros, riesgo éste que corre la autoevaluación y que dificulta unas acciones de mejora más concretas.

Uno de los puntos débiles que se han detectado en las evaluaciones de Deusto y Granada, es la ausencia de comentarios referidos a la creación de los comités de evaluación que han llevado a cabo estos procesos. Esto dificulta el tener un conocimiento más preciso sobre cómo se ha desarrollado la labor evaluativa, es decir, todos los informes deberían haber presentado la constitución de su equipo de trabajo, la planificación de cómo iban a realizar la autoevaluación, y las deficiencias que han encontrado una vez finalizado el proceso. Del mismo modo, y exceptuando a la Facultad de Granada, ninguno de los otros informes han dedicado un apartado concreto a la metodología que han utilizado para obtener la información requerida. A pesar de esto, el análisis de los informes muestra que se ha utilizado tanto metodología cualitativa como cuantitativa. Las técnicas han sido diversas, destacando la encuesta, las entrevistas, el uso de fuentes secundarias y los grupos de discusión (Granada).

Otro de los puntos negativos detectados tras el análisis de los informes de autoevaluación, es la ausencia de unos objetivos claros y específicos, tanto en la enseñanza y en la investigación, como sobre todo en la gestión. Esto supone un obstáculo para la evaluación, pues a pesar de no ser este un proceso basado en objetivos, siempre es recomendable conocer los propósitos de las instituciones para saber qué evaluar. Además, la existencia de 
unas metas prefijadas facilita no sólo la labor del equipo evaluador, sino que ayuda al desarrollo de un programa de evaluación de carácter formativo o continuo.

La excesiva tendencia a ponderar el estudio de las relaciones internas es un punto débil que aparece en los tres informes, olvidando en muchos casos las externas. Este problema puede estar motivado por las características propias de la autoevaluación y de los responsables de llevarlas a cabo, los cuales al pertenecer a la propia titulación ponderan más el estudio del centro que la relación que éste tiene con el entorno que le rodea, y no le prestan demasiada consideración a la dependencia que existe entre el mercado de trabajo y sus licenciados, o a los convenios entre las empresas e instituciones públicas y los departamentos.
El análisis metodológico de los distintos planes de evaluación no sólo sirve para detectar si lo establecido por el PNECU es seguido por los distintos centros, sino que ayuda a enriquecer la metodología utilizada en las distintas evaluaciones llevadas a cabo en facultades que imparten la misma licenciatura. Todos los comités han aportado algunos indicadores que no aparecen en la Guía de Evaluación, y que han sido considerados necesarios para conocer la realidad universitaria. Igualmente, la necesidad de mejorar la calidad obliga a todos los expertos en materia evaluativa a buscar un necesario consenso entre los indicadores y la metodología más apropiada para medir este concepto. Por todo ello, desde estas líneas se anima a realizar trabajos que identifiquen indicadores para medir de manera adecuada la calidad en la enseñanza universitaria.
* Profesor Titular de Sociología en la Facultad de Ciencias Políticas y Sociología de Granada.

${ }^{*}$ Investigador del Departamento de Sociología de la Facultad de Ciencias y Sociología de Granada.
${ }^{* * *}$ Profesora Titular de Ciencias Políticas en la Facultad de Ciencias y Sociología de Granada.

\section{Bibliografia}

Alemay, R. (2000) Size, J. (1991) "Indicadores de calidad docente ¡café para todos?" en Indicadores en la Universidad: Información y decisiones. Edita MEC. (pp 301-311).

ArÉVAlo, J. ECHEVERría, Ma José y MarTí, S (2000) "La gestión de las bibliotecas universitarias: indicadores para su evaluación” en Indicadores en la Universidad: Información y decisiones. Edita MEC. (pp 479-491)

Bol, A y Tricio, V. (2000) "Una experiencia departamental en evaluación de la calidad y adaptación de los indicadores, para la investigación, la enseñanza y gestión”. En Indicadores en la Universidad: Información y decisiones. Edita MEC. (pp 231-251).

Consejo de Universidades (1996). Guía de evaluación del Plan Nacional de Evaluación de la calidad de las Universidades. Madrid.

CuEnin, S. (1987). "The use of performance indicators in universities: An international survey". International Journal of Management in Higher Education, 11, 2, $117-139$

De Miguel, M. (1991) "La evaluación de las instituciones universitarias". Granada. Ponencia IV Jornadas de Didáctica Universitaria. Universidad de Granada.

De Miguel, M. (2000) "La evaluación de la enseñanza. Propuestas de indicadores para las titulaciones" en Indicadores en la Universidad: Información y decisiones. Edita MEC. (pp 413-431)

Dobson, I. Sharma, R. y Hayddon, A (1998). "Undergraduate intakes in Australia: before and after”. Higher Education Management, 10 (1), 43-54.

Dochy, F.J.R.C. (1991) La evaluación de las instituciones universitarias. Consejo de Universidades, Madrid.

Frackmann, E (1991) "Lecciones que deben aprenderse de una década de discusiones sobre indicadores de rendimiento" en DE Miguel, M. La evaluación de las instituciones universitarias, Consejo de universidades, Madrid.
GraO, J y Winter, R. (2000) "Indicadores para la calidad y calidad de los indicadores" en Indicadores en la Universidad: Información y decisiones. Edita MEC. (pp. 81-87)

House, E.R. y Yeager (1979). "Coherence and credibility: The esthetics of evaluation", en Educational evaluation and policy analysis, 1,5.

IgLESIAS DE UsSEL, J (2000) "La universidad en la sociedad del conocimiento" en Evaluación y calidad en las organizaciones públicas. Ed. INAP, Madrid

OrTega y GASSET (1976) Misión de la Universidad: y otros ensayos afines. Revista de Occidente. Madrid

Pérez Vera. E. (1991). Actas de la jornada: Planes de estudio y calidad de la enseñanza universitaria. Universidad Politécnica de Madrid (pp. 163-174).

Rey GarCía, A. (1988) Como gestionar la calidad en las universidades, el modelo europeo de excelencia universitaria. Ed Club de Gestión de Calidad. Madrid

SCRIVEN, M.S. (1967). "Evaluation bias and its control". Ocassional Paper Series. N4, Universidad de Western Michigan. Centro de Evaluación.

Simons (1985). "Suggestions for a school self evaluation based on democratic principies", en R. Mccormick et. (Eds.) Calling education to account. London: Heinemann- The Open University Press.

SIZER, J. (1991) "Comités de financiación e indicadores de rendimiento en la evolución de la calidad en el Reino Unido", en La evaluación de las instituciones universitarias. Consejo de Universidades. Madrid.

STUFFLEBEAM, D.L. (1981). Standards for Evaluations of Educational Programmes, Projects, and Materials, Joint Committee on Standars for Educational Evaluation. McGrawHill, Nueva York.

StufFlebeAm, D.L. y SHINKFIELD, A.J. (1987). Evaluación sistemática: guia teórica y práctica. Paidós-MEC, Madrid. 
Tricio, V; BOL, A; VILORA, R. (2000) "Una experiencia departamental en evaluación de la calidad y adaptación de los indicadores, para investigación enseñanza y gestión" En Indicadores en la Universidad: Información y decisiones. Edita MEC. (pp 231-251)

Trinidad, A. (1995). La evaluación de instituciones educativas. El análisis práctico de la Facultad de CC. Politicas y Sociología de la Universidad de Granada. Servicio de Publicaciones de la Universidad de Granada.

TyLeR, R.W. (1967). "Changing concepts of educational evaluation". En R.E. STAKE (ed). Perspectives of curriculum evaluation (vol. I), New York: Rand McNally.
VIDAL, J. (2000) "Indicadores de rendimiento para las universidades españolas: necesidades y disponibilidad". En Indicadores en la Universidad: Información y decisiones. Edita MEC. (pp. 7-19).

ZORRILA, R. (2000) "Indicadores en los servicios universitarios", en Indicadores en la Universidad: Información y decisiones. Edita MEC. (pp 457-467) 
